\title{
CONSTANT-SIGN SOLUTIONS OF A SYSTEM OF VOLTERRA INTEGRAL EQUATIONS IN ORLICZ SPACES
}

\author{
RAVI P. AGARWAL, DONAL O'REGAN, AND PATRICIA J. Y. WONG
}

Communicated by Charles Groetsch

This paper is dedicated to Professor Zuhair Nashed in recognition of his contributions to the field of integral and operator equations.

ABSTRACT. We consider the following system of Volterra intergral equations

$$
\begin{gathered}
u_{i}(t)=\int_{0}^{t} g_{i}(t, s) f_{i}\left(s, u_{1}(s), u_{2}(s), \cdots, u_{n}(s)\right) d s, \\
\text { a.e. } t \in[0, T], 1 \leq i \leq n .
\end{gathered}
$$

Criteria are offered for the existence of one and more constantsign solutions $u=\left(u_{1}, u_{2}, \cdots, u_{n}\right)$ of the system in $L^{p}$ and the Orlicz spaces. We say $u$ is of constant sign if for each $1 \leq i \leq n, \theta_{i} u_{i}(t) \geq 0$ for a.e. $t \in[0, T]$, where $\theta_{i} \in\{1,-1\}$ is fixed.

1. Introduction. In this paper we shall consider the system of Volterra integral equations

$$
\begin{gathered}
u_{i}(t)=\int_{0}^{t} g_{i}(t, s) f_{i}\left(s, u_{1}(s), u_{2}(s), \cdots, u_{n}(s)\right) d s, \\
\text { a.e. } t \in[0, T], 1 \leq i \leq n .
\end{gathered}
$$

Throughout, let $u=\left(u_{1}, u_{2}, \cdots, u_{n}\right)$. We are interested in establishing the existence of one and more solutions $u$ of the system (1.1) in

\footnotetext{
2000 AMS Mathematics subject classification. Primary 45B05, 45G15, 45M20.

Keywords and phrases. Constant-sign solutions, system of Volterra integral equations, Orlicz space.

Received by the editors on November 2, 2006, and in revised form on February 7, 2007.

DOI:10.1216/JIE-2008-20-3-337 Copyright (C)2008 Rocky Mountain Mathematics Consortium
} 
Orlicz spaces, i.e., for each $1 \leq i \leq n, u_{i}$ is in the Orlicz space $L_{\phi}$. Moreover, we are interested in constant-sign solutions $u$, by which we mean $\theta_{i} u_{i}(t) \geq 0$ for a.e. $t \in[0, T]$ and $1 \leq i \leq n$, where $\theta_{i} \in\{1,-1\}$ is fixed. Note that positive solution is a special case of constant-sign solution when $\theta_{i}=1$ for $1 \leq i \leq n$.

We shall consider the existence of solutions in $L^{p}(p \geq 1)$ spaces first, since we can obtain more general results here and also the analysis will motivate the ideas later in obtaining existence results in Orlicz spaces. We remark that those results obtained for $L^{p}$ spaces invariably assume a polynomial type restriction (in $u$ ) on the nonlinearity $f_{i}(t, u)$ (see condition (C2) later). On the other hand, seeking solutions in other Orlicz spaces will lead to restrictions that are not of polynomial type (see condition (H4) later), and hence will allow us to consider new classes of equations. The main tools employed in this paper are the Leray-Schauder alternative and the Krasnosel'skii's fixed point theorem.

The existence of multiple constant-sign solutions of $(1.1)$ in $(C[0, T])^{n}$ has recently been tackled in [7]. However, to the knowledge of the authors, there is very little literature on the existence of solutions of Volterra integral equations in $L^{p}$ spaces, the only paper in this area [15] applies Krasnosel'skii's fixed point theorem to obtain a positive solution of

$$
y(t)=\int_{0}^{t} g(t, s) f(s, y(s)) d s, \quad \text { a.e. } t \in[0, T] .
$$

Moreover, to date very little work has been done on the existence of solutions of Volterra integral equations in Orlicz spaces. Hence, our present work not only generalizes and extends those of [15] to (i) systems, and (ii) the existence of multiple constant-sign solutions through the use of two fixed point theorems, but also investigates the existence of multiple constant-sign solutions in Orlicz spaces, which is totally new in the literature.

We note that, on the other hand, a lot of work has been done on the existence of solutions of Fredholm integral equations of the form

$$
y(t)=\int_{0}^{T} g(t, s) f(s, y(s)) d s, \quad t \in[0, T]
$$

Mostly solutions are sought in $C[0, T]$ or $L^{p}[0, T]$ with $p>1[1,10,11$, 13], whereas solutions in Orlicz spaces are tackled in $[14,16,17,19$, 
20]. The more recent investigation on the existence of constant-sign solutions of systems of Fredholm integral equations in continuous, $L^{p}$ and Orlicz spaces can be found in Agarwal, O'Regan and Wong [2-6].

The plan of the paper is as follows. The existence of constant-sign solutions of (1.1) in $L^{p}$ and Orlicz spaces will be tackled respectively in Sections 3 and 4 . Examples are also presented to illustrate the usefulness of the results obtained.

2. Preliminaries The following two theorems will be needed to establish the main results later. The first theorem is known as the LeraySchauder alternative and the second is usually called Krasnosel'skii's fixed point theorem in a cone.

Theorem 2.1. [1] Let $B$ be a Banach space with $E \subseteq B$ closed and convex. Assume $U$ is a relatively open subset of $E$ with $0 \in U$ and $S: \bar{U} \rightarrow E$ is a continuous and compact map. Then either

(a) $S$ has a fixed point in $\bar{U}$, or

(b) there exists $u \in \partial U$ and $\lambda \in(0,1)$ such that $u=\lambda S u$.

Theorem 2.2. [12] Let $B=(B,\|\cdot\|)$ be a Banach space, and let $C \subset B$ be a cone in $B$. Assume $\Omega_{1}, \Omega_{2}$ are open subsets of $B$ with $0 \in \Omega_{1}, \bar{\Omega}_{1} \subset \Omega_{2}$, and let $S: C \cap\left(\bar{\Omega}_{2} \backslash \Omega_{1}\right) \rightarrow C$ be a continuous and completely continuous operator such that, either

(a) $\|S u\| \leq\|u\|, u \in C \cap \partial \Omega_{1}$, and $\|S u\| \geq\|u\|, u \in C \cap \partial \Omega_{2}$, or

(b) $\|S u\| \geq\|u\|, u \in C \cap \partial \Omega_{1}$, and $\|S u\| \leq\|u\|, u \in C \cap \partial \Omega_{2}$.

Then, $S$ has a fixed point in $C \cap\left(\bar{\Omega}_{2} \backslash \Omega_{1}\right)$.

3. Existence in $L^{p}$ Space In this section, we consider the system (1.1) where, for each $1 \leq i \leq n, g_{i}(t, s)$ is a real-valued function for a.e. $t \in[0, T]$ and a.e. $s \in[0, t]$, and $f_{i}(t, u)$ is also a real-valued function for a.e. $t \in[0, T]$ and all $u \in \mathbb{R}^{n}$. We shall establish the existence of a constant-sign solution $u \in\left(L^{p}[0, T]\right)^{n}=$ $L^{p}[0, T] \times L^{p}[0, T] \times \cdots \times L^{p}[0, T](n$ times) of $(1.1)$ where $p \geq 1$. Throughout this section, let $\theta_{i} \in\{1,-1\}, 1 \leq i \leq n$ be fixed, also let the integers $p_{1}$ and $p_{2}$ be such that 


$$
1 \leq p_{1} \leq p<\infty \quad \text { and } \quad \frac{1}{p_{1}}+\frac{1}{p_{2}}=1 .
$$

Let the Banach space $B=\left(L^{p}[0, T]\right)^{n}$ be equipped with the norm

$$
\|u\|_{p}=\max _{1 \leq i \leq n}\left(\int_{0}^{T}\left|u_{i}(t)\right|^{p} d t\right)^{\frac{1}{p}}=\max _{1 \leq i \leq n}\left|u_{i}\right|_{p}
$$

where we let $\left|u_{i}\right|_{p}=\left(\int_{0}^{T}\left|u_{i}(t)\right|^{p} d t\right)^{\frac{1}{p}}, 1 \leq i \leq n$.

To begin our discussion, let the operator $S: B \rightarrow B$ be defined by

$$
S u(t)=\left(S_{1} u(t), S_{2} u(t), \cdots, S_{n} u(t)\right), \quad \text { a.e.t } \in[0, T]
$$

where

$$
S_{i} u(t)=\int_{0}^{t} g_{i}(t, s) f_{i}(s, u(s)) d s, \quad \text { a.e.t } \in[0, T], \quad 1 \leq i \leq n .
$$

Clearly, a fixed point of the operator $S$ is a solution of the system (1.1).

Our first two lemmas show that $S: B \rightarrow B$ is well defined, and is continuous and completely continuous.

Lemma 3.1. Assume

(C1) for each $1 \leq i \leq n, f_{i}:[0, T] \times \mathbb{R}^{n} \rightarrow \mathbb{R}$ is a Carathéodory function, i.e.,

(i) the map $t \mapsto f_{i}(t, u)$ is measurable for all $u \in \mathbb{R}^{n}$,

(ii) the map $u \mapsto f_{i}(t, u)$ is continuous for almost all $t \in[0, T]$;

(C2) for each $1 \leq i \leq n$, there exists a function $b_{i} \in L^{p_{2}}[0, T]$ and constants $c_{i j}>0,1 \leq j \leq n$ such that

$$
\left|f_{i}(t, u)\right| \leq b_{i}(t)+\sum_{j=1}^{n} c_{i j}\left|u_{j}\right|^{\frac{p}{p_{2}}}, \quad \text { a.e. } t \in[0, T], \quad \text { all } u \in \mathbb{R}^{n}
$$


(C3) for each $1 \leq i \leq n$, the map $(t, s) \rightarrow g_{i}(t, s)$ is measurable, and $g_{i}(t, s)>0$ for a.e. $t \in[0, T]$, a.e. $s \in[0, t]$;

(C4) for each $1 \leq i \leq n$,

$$
\left[\int_{0}^{T}\left(\int_{s}^{T}\left|g_{i}(t, s)\right|^{p} d t\right)^{\frac{p_{1}}{p}} d s\right]^{\frac{1}{p_{1}}} \equiv M_{i}<\infty .
$$

Then, the operator $S: B \rightarrow B$ is well defined.

Proof. For each $1 \leq i \leq n$, let

$$
g_{i}^{*}(t, s)= \begin{cases}g_{i}(t, s), & 0 \leq s \leq t \leq T \\ 0, & 0 \leq t<s \leq T .\end{cases}
$$

First, using Tonelli's Theorem and the fact that if a function $y$ is measurable, then so is $|y|^{p}$, we see that for each $1 \leq i \leq n$,

$$
\left(\int_{0}^{T}\left|g_{i}^{*}(t, s)\right|^{p_{1}} d s\right)^{\frac{p}{p_{1}}}=\left(\int_{0}^{t}\left|g_{i}(t, s)\right|^{p_{1}} d s\right)^{\frac{p}{p_{1}}}
$$

is a measurable function of $t$ on $[0, T]$.

Applying the integral version of Minkowski's inequality [21, p.143], we obtain, noting $\frac{p}{p_{1}} \geq 1$ and also (C4),

$$
\begin{aligned}
\int_{0}^{T}\left(\int_{0}^{t}\left|g_{i}(t, s)\right|^{p_{1}} d s\right)^{\frac{p}{p_{1}}} d t & =\int_{0}^{T}\left(\int_{0}^{T}\left|g_{i}^{*}(t, s)\right|^{p_{1}} d s\right)^{\frac{p}{p_{1}}} d t \\
& \leq\left[\int_{0}^{T}\left(\int_{0}^{T}\left|g_{i}^{*}(t, s)\right|^{p} d t\right)^{\frac{p_{1}}{p}} d s\right]^{\frac{p}{p_{1}}} \\
& =\left[\int_{0}^{T}\left(\int_{s}^{T}\left|g_{i}(t, s)\right|^{p} d t\right)^{\frac{p_{1}}{p}} d s\right]^{\frac{p}{p_{1}}} \\
& =M_{i}^{p}, 1 \leq i \leq n .
\end{aligned}
$$

Hence, $\left(\int_{0}^{t}\left|g_{i}(t, s)\right|^{p_{1}} d s\right)^{\frac{p}{p_{1}}}$ is integrable on $[0, T]$.

Let $u \in\left(L^{p}[0, T]\right)^{n}$. In view of $(\mathrm{C} 2)$, it is clear that 


$$
\begin{aligned}
\left(\int_{0}^{T}\left|f_{i}(t, u(t))\right|^{p_{2}} d t\right)^{\frac{1}{p_{2}}} & \leq\left|b_{i}\right|_{p_{2}}+\sum_{j=1}^{n} c_{i j}\left(\int_{0}^{T}\left|u_{j}(t)\right|^{p} d t\right)^{\frac{1}{p_{2}}} \\
& \equiv L_{i}<\infty, 1 \leq i \leq n
\end{aligned}
$$

Therefore,

$$
f_{i}(t, u) \in L^{p_{2}}[0, T] \text { for } u \in\left(L^{p}[0, T]\right)^{n}, \quad 1 \leq i \leq n .
$$

Now, for each $1 \leq i \leq n$, we use Hölder's inequality, (3.4) and (3.5) to get

$$
\begin{aligned}
\int_{0}^{T}\left|S_{i} u(t)\right|^{p} d t & \leq \int_{0}^{T}\left[\int_{0}^{t}\left|g_{i}(t, s)\right| \cdot\left|f_{i}(s, u(s))\right| d s\right]^{p} d t \\
& \leq \int_{0}^{T}\left[\left(\int_{0}^{t}\left|g_{i}(t, s)\right|^{p_{1}} d s\right)^{\frac{1}{p_{1}}}\left(\int_{0}^{T}\left|f_{i}(s, u(s))\right|^{p_{2}} d s\right)^{\frac{1}{p_{2}}}\right]^{p} d t \\
& \leq M_{i}^{p} L_{i}^{p} .
\end{aligned}
$$

This leads to

$$
\left|S_{i} u\right|_{p} \leq M_{i} L_{i}<\infty \text { for } u \in\left(L^{p}[0, T]\right)^{n}, \quad 1 \leq i \leq n .
$$

Hence, $S_{i} u \in L^{p}[0, T], 1 \leq i \leq n$ and so $S u \in\left(L^{p}[0, T]\right)^{n}$. This shows that $S:\left(L^{p}[0, T]\right)^{n} \rightarrow\left(L^{p}[0, T]\right)^{n}$ is well defined.

Lemma 3.2. Let $(\mathrm{C} 1)-(\mathrm{C} 4)$ hold. Then, the operator $S: B \rightarrow B$ is continuous and completely continuous.

Proof. We shall show that $S_{i}, 1 \leq i \leq n$ is continuous and completely continuous. For each $1 \leq i \leq n$, we observe that the operator $S_{i}:\left(L^{p}[0, T]\right)^{n} \rightarrow L^{p}[0, T]$ can be written as

$$
S_{i}=A_{i} F_{i}
$$


where $F_{i}:\left(L^{p}[0, T]\right)^{n} \rightarrow L^{p_{2}}[0, T]$ and $A_{i}: L^{p_{2}}[0, T] \rightarrow L^{p}[0, T]$

are respectively defined by

$$
F_{i} u(t)=f_{i}(t, u(t)), \quad \text { a.e. } t \in[0, T]
$$

and

$$
A_{i} x(t)=\int_{0}^{t} g_{i}(t, s) x(s) d s, \quad \text { a.e. } t \in[0, T] .
$$

Note that $F_{i}:\left(L^{p}[0, T]\right)^{n} \rightarrow L^{p_{2}}[0, T]$ is well defined by (3.6). Further, for $x \in L^{p_{2}}[0, T]$, using (3.4) we have

$$
\begin{aligned}
\int_{0}^{T}\left|A_{i} x(t)\right|^{p} d t & \leq \int_{0}^{T}\left[\int_{0}^{t}\left|g_{i}(t, s)\right| \cdot|x(s)| d s\right]^{p} d t \\
& \leq \int_{0}^{T}\left[\left(\int_{0}^{t}\left|g_{i}(t, s)\right|^{p_{1}} d s\right)^{\frac{1}{p_{1}}}\left(\int_{0}^{T}|x(s)|^{p_{2}} d s\right)^{\frac{1}{p_{2}}}\right]^{p} d t \\
& \leq M_{i}^{p}|x|_{p_{2}}^{p} .
\end{aligned}
$$

Therefore,

$$
\left|A_{i} x\right|_{p} \leq M_{i}|x|_{p_{2}}<\infty \quad \text { for } \quad x \in L^{p_{2}}[0, T]
$$

This shows that $A_{i}: L^{p_{2}}[0, T] \rightarrow L^{p}[0, T]$ is well defined. Indeed, it has been noted earlier that $S_{i}=A_{i} F_{i}:\left(L^{p}[0, T]\right)^{n} \rightarrow L^{p}[0, T], 1 \leq i \leq n$, is well defined (see (3.7)).

By a result of Krasnosel'skii [13, p.22, p.27], it follows that $F_{i}$ : $\left(L^{p}[0, T]\right)^{n} \rightarrow L^{p_{2}}[0, T]$ is continuous and bounded. Thus, to prove that $S_{i}$ is continuous and completely continuous, it suffices to show that $A_{i}$ is continuous and completely continuous.

First, we shall prove that $A_{i}$ is continuous. Let $x_{m} \rightarrow x$ in $L^{p_{2}}[0, T]$, i.e., $\lim _{m \rightarrow \infty}\left|x_{m}-x\right|_{p_{2}}=0$. Applying Hölder's inequality and (3.4), we find 


$$
\begin{aligned}
\int_{0}^{T}\left|A_{i} x_{m}(t)-A_{i} x(t)\right|^{p} d t & \leq \int_{0}^{T}\left[\int_{0}^{t}\left|g_{i}(t, s)\right| \cdot\left|x_{m}(s)-x(s)\right| d s\right]^{p} d t \\
& \leq \int_{0}^{T}\left[\left(\int_{0}^{t}\left|g_{i}(t, s)\right|^{p_{1}} d s\right)^{\frac{1}{p_{1}}}\right. \\
& \left.\left(\int_{0}^{T}\left|x_{m}(s)-x(s)\right|^{p_{2}} d s\right)^{\frac{1}{p_{2}}}\right]^{p} d t \\
& \leq M_{i}^{p}\left|x_{m}-x\right|_{p_{2}}^{p}
\end{aligned}
$$

or

$$
\left|A_{i} x_{m}-A_{i} x\right|_{p} \leq M_{i}\left|x_{m}-x\right|_{p_{2}} \rightarrow 0 \quad \text { as } \quad m \rightarrow \infty .
$$

Hence, $A_{i}$ is continuous.

Next, we shall show that $A_{i}$ is completely continuous. Let $\Omega$ be a bounded set in $L^{p_{2}}[0, T]$, i.e., there exists a constant $K>0$ such that

$$
|x|_{p_{2}} \leq K, \quad x \in \Omega .
$$

We shall use the Riesz Compactness Criteria [18, p.10] to show that $A_{i} \Omega$ is relatively compact. The first condition in the criteria is to have $A_{i} \Omega$ bounded in $L^{p}[0, T]$. This is satisfied in view of (3.11) and (3.12). The second condition in the criteria is to have the following satisfied

(3.13) $\int_{0}^{T}\left|A_{i} x(t+h)-A_{i} x(t)\right|^{p} d t \rightarrow 0$ as $h \rightarrow 0$, uniformly for $x \in \Omega$.

In fact, applying Hölder's inequality, the integral version of Minkowski's inequality [21, p.143] and (3.12), we find for $x \in \Omega$, 


$$
\begin{aligned}
& \int_{0}^{T}\left|A_{i} x(t+h)-A_{i} x(t)\right|^{p} d t \\
\leq & \int_{0}^{T}\left[\int_{0}^{t}\left|g_{i}(t+h, s)-g_{i}(t, s)\right| \cdot|x(s)| d s\right. \\
& \left.+\int_{t}^{t+h}\left|g_{i}(t+h, s)\right| \cdot|x(s)| d s\right]^{p} d t \\
= & \int_{0}^{T}\left[\int_{0}^{T}\left|g_{i}^{*}(t+h, s)-g_{i}^{*}(t, s)\right| \cdot|x(s)| d s\right]^{p} d t \\
\leq & \int_{0}^{T}\left[\left(\int_{0}^{T}\left|g_{i}^{*}(t+h, s)-g_{i}^{*}(t, s)\right|^{p_{1}} d s\right)^{\frac{1}{p_{1}}}\left(\int_{0}^{T}|x(s)|^{p_{2}} d s\right)^{\frac{1}{p_{2}}}\right]^{p} d t \\
= & |x|_{p_{2}}^{p} \int_{0}^{T}\left(\int_{0}^{T}\left|g_{i}^{*}(t+h, s)-g_{i}^{*}(t, s)\right|^{p_{1}} d s\right)^{\frac{p}{p_{1}}} d t \\
\leq & K^{p}\left[\int_{0}^{T}\left(\int_{0}^{T}\left|g_{i}^{*}(t+h, s)-g_{i}^{*}(t, s)\right|^{p} d t\right)^{\frac{p_{1}}{p}} d s\right]^{\frac{p}{p_{1}}} .
\end{aligned}
$$

Now noting (3.4) and the fact that translates of $L^{p}$ functions $(1 \leq$ $p<\infty)$ are continuous in norm, we see that (3.13) holds. Hence, the Riesz Compactness Criteria are fulfilled and we have shown that $A_{i} \Omega$ is relatively compact, so $A_{i}$ is completely continuous. The proof is complete.

We are now ready to employ Theorem 2.1 to get a general existence result in $\left(L^{p}[0,1]\right)^{n}$.

Theorem 3.1. Let $(\mathrm{C} 1)-(\mathrm{C} 4)$ hold. Assume there is a positive constant $M_{0}$, independent of $\lambda$, with

$$
\|u\|_{p} \neq M_{0}
$$

for any solution $u \in\left(L^{p}[0, T]\right)^{n}$ of the system 


$$
u(t)=\lambda S u(t), \quad \text { a.e. } t \in[0, T]
$$

where $0<\lambda<1$. Then, the system (1.1) has a solution $u^{*} \in$ $\left(L^{p}[0, T]\right)^{n}$ with $\left\|u^{*}\right\|_{p} \leq M_{0}$.

Proof. By Lemma 3.2, (C1)-(C4) guarantee that $S: B \rightarrow B$ is continuous and completely continuous. In the context of Theorem 2.1, let

$$
U=\left\{u \in B \mid\|u\|_{p}<M_{0}\right\} .
$$

Since $\|u\|_{p} \neq M_{0}$, where $u$ is any solution of (3.15), we cannot have conclusion (b) of Theorem 2.1, hence conclusion (a) of Theorem 2.1 must hold, i.e., the system (1.1) has a solution $u^{*} \in \bar{U}$ with $\left\|u^{*}\right\|_{p} \leq M_{0}$.

口

The next result applies Theorem 3.1 to get the existence of a constantsign solution in $\left(L^{p}[0,1]\right)^{n}$.

Theorem 3.2. Let $(\mathrm{C} 1)-(\mathrm{C} 4)$ hold. Assume

(C5) for each $1 \leq i \leq n$,

$$
\theta_{i} f_{i}(t, u) \geq 0, \text { a.e. } t \in[0, T], \quad \text { all } u \in \tilde{K}
$$

where

$$
\tilde{K}=\left\{u \in B \mid \text { for each } 1 \leq i \leq n, \theta_{i} u_{i}(t) \geq 0 \text { for a.e. } t \in[0, T]\right\} ;
$$

(C6) there exists $\alpha>0$ such that for each $1 \leq i \leq n$,

$$
M_{i} \psi_{i}(\alpha)<\alpha
$$

where $\psi_{i}:[0, \infty) \rightarrow[0, \infty)$ is defined by

$$
\psi_{i}(x)=\left|b_{i}\right|_{p_{2}}+(x)^{\frac{p}{p_{2}}} \sum_{j=1}^{n} c_{i j} .
$$

Then, the system (1.1) has at least one constant-sign solution $u \in$ $\left(L^{p}[0, T]\right)^{n}$ such that $\|u\|_{p}<\alpha$. 
Proof. To begin, we consider the system

$$
\begin{gathered}
u_{i}(t)=\int_{0}^{t} g_{i}(t, s) \hat{f}_{i}\left(s, u_{1}(s), u_{2}(s), \cdots, u_{n}(s)\right) d s, \\
\text { a.e. } t \in[0, T], \quad 1 \leq i \leq n
\end{gathered}
$$

where $\hat{f}_{i}:[0, T] \times \mathbb{R}^{n} \rightarrow \mathbb{R}$ is defined by

$$
\begin{aligned}
\hat{f}_{i}\left(t, u_{1}, u_{2}, \cdots, u_{n}\right)= & f_{i}\left(t, \theta_{1}\left|u_{1}\right|, \theta_{2}\left|u_{2}\right|, \cdots, \theta_{n}\left|u_{n}\right|\right), \\
& 1 \leq i \leq n
\end{aligned}
$$

Note that $\left(\theta_{1}\left|u_{1}\right|, \theta_{2}\left|u_{2}\right|, \cdots, \theta_{n}\left|u_{n}\right|\right) \in \tilde{K}$.

We shall prove that (3.16) has a solution. For this, we consider the system

$(3.18)_{\lambda} \quad u_{i}(t)=\lambda \int_{0}^{t} g_{i}(t, s) \hat{f}_{i}(s, u(s)) d s, \quad$ a.e. $t \in[0, T], \quad 1 \leq i \leq n$

where $0<\lambda<1$. Let $u \in\left(L^{p}[0, T]\right)^{n}$ be any solution of (3.18). We shall show that

$$
\|u\|_{p} \neq \alpha
$$

then by Theorem 3.1 it follows that (3.16) has a solution.

Now, using (3.17), (C3) and (C5), we get

$$
\begin{aligned}
\theta_{i} u_{i}(t) & =\lambda \int_{0}^{t} g_{i}(t, s) \theta_{i} \hat{f}_{i}\left(s, u_{1}(s), u_{2}(s), \cdots, u_{n}(s)\right) d s \\
& =\lambda \int_{0}^{t} g_{i}(t, s) \theta_{i} f_{i}\left(s, \theta_{1}\left|u_{1}(s)\right|, \theta_{2}\left|u_{2}(s)\right|, \cdots, \theta_{n}\left|u_{n}(s)\right|\right) d s \geq 0, \\
& \text { a.e. } t \in[0, T], 1 \leq i \leq n
\end{aligned}
$$

which means that

$$
\left|u_{i}(t)\right|=\theta_{i} u_{i}(t) \text {, a.e. } t \in[0, T], \quad 1 \leq i \leq n .
$$


Moreover, it is clear that for each $1 \leq i \leq n$,

$$
\begin{aligned}
& \int_{0}^{T}\left|u_{i}(t)\right|^{p} d t \\
\leq & \int_{0}^{T}\left[\int_{0}^{t}\left|g_{i}(t, s)\right| \cdot\left|f_{i}\left(s, \theta_{1}\left|u_{1}(s)\right|, \theta_{2}\left|u_{2}(s)\right|, \cdots, \theta_{n}\left|u_{n}(s)\right|\right)\right| d s\right]^{p} d t .
\end{aligned}
$$

Using the same argument as in getting (3.7), we find

$$
\begin{aligned}
\left|u_{i}\right|_{p} & \leq M_{i}\left[\left|b_{i}\right|_{p_{2}}+\sum_{j=1}^{n} c_{i j}\left(\left|u_{j}\right|_{p}\right)^{\frac{p}{p_{2}}}\right] \\
& \leq M_{i}\left[\left|b_{i}\right|_{p_{2}}+\sum_{j=1}^{n} c_{i j}\left(\|u\|_{p}\right)^{\frac{p}{p_{2}}}\right]=M_{i} \psi_{i}\left(\|u\|_{p}\right), \quad 1 \leq i \leq n .
\end{aligned}
$$

It follows that

$$
\|u\|_{p} \leq \max _{1 \leq j \leq n} M_{j} \psi_{j}\left(\|u\|_{p}\right)
$$

Noting (3.21) and (C6), we conclude that $\|u\|_{p} \neq \alpha$. Hence, (3.19) is proved.

It now follows from Theorem 3.1 that the system (3.16) has a solution $u^{*} \in\left(L^{p}[0, T]\right)^{n}$ with $\left\|u^{*}\right\|_{p} \leq \alpha$, and

$$
\begin{gathered}
u_{i}^{*}(t)=\int_{0}^{t} g_{i}(t, s) \hat{f}_{i}\left(s, u_{1}^{*}(s), u_{2}^{*}(s), \cdots, u_{n}^{*}(s)\right) d s, \\
\text { a.e. } t \in[0, T], 1 \leq i \leq n .
\end{gathered}
$$

Using a similar argument as above, it can be easily seen that

$$
\left|u_{i}^{*}(t)\right|=\theta_{i} u_{i}^{*}(t) \text {, a.e. } t \in[0, T], \quad 1 \leq i \leq n
$$

and

$$
\left\|u^{*}\right\|_{p} \neq \alpha
$$


Therefore, $u^{*}$ is of constant sign and $\left\|u^{*}\right\|_{p}<\alpha$. Further, using (3.17) and (3.22), we have for a.e. $t \in[0, T]$ and each $1 \leq i \leq n$,

$$
\begin{aligned}
u_{i}^{*}(t) & =\int_{0}^{t} g_{i}(t, s) \hat{f}_{i}\left(s, u_{1}^{*}(s), u_{2}^{*}(s), \cdots, u_{n}^{*}(s)\right) d s \\
& =\int_{0}^{t} g_{i}(t, s) f_{i}\left(s, \theta_{1}\left|u_{1}^{*}(s)\right|, \theta_{2}\left|u_{2}^{*}(s)\right|, \cdots, \theta_{n}\left|u_{n}^{*}(s)\right|\right) d s \\
& =\int_{0}^{t} g_{i}(t, s) f_{i}\left(s, \theta_{1}^{2} u_{1}^{*}(s), \theta_{2}^{2} u_{2}^{*}(s), \cdots, \theta_{n}^{2} u_{n}^{*}(s)\right) d s \\
& =\int_{0}^{t} g_{i}(t, s) f_{i}\left(s, u_{1}^{*}(s), u_{2}^{*}(s), \cdots, u_{n}^{*}(s)\right) d s .
\end{aligned}
$$

Hence, $u^{*}$ is in fact a solution of (1.1). The proof is complete.

Remark 3.1. By examining the proof of Theorem 3.2, we realize that the function $\psi_{i}$ (appeared in (C6)) must be such that

$$
\left(\int_{0}^{T}\left|f_{i}(t, u(t))\right|^{p_{2}} d t\right)^{\frac{1}{p_{2}}} \leq \psi_{i}\left(\|u\|_{p}\right) .
$$

Therefore, $\psi_{i}$ can be defined differently from the one in (C6), so long as it satisfies the above inequality.

Remark 3.2. If $\frac{p}{p_{2}}<1$, then the existence of $\alpha$ in (C6) is guaranteed.

We have so far used the Leray-Schauder alternative (Theorem 2.1) to obtain existence criteria in $\left(L^{p}[0,1]\right)^{n}$. We shall next apply Krasnosel' skii's fixed point theorem (Theorem 2.2) to get further existence results in $\left(L^{p}[0,1]\right)^{n}$.

Define a cone in $B$ as

$$
\begin{aligned}
& =\left\{u \in B \mid \text { for each } 1 \leq i \leq n, \theta_{i} u_{i}(t) \geq a(t)\|u\|_{p} \text { for a.e. } t \in[0, T]\right\} .
\end{aligned}
$$

Here we assume

$$
a(t)>0 \text {, a.e. } t \in[0, T], \quad a \in L^{p}[0, T] \quad \text { and } \quad\|a\|_{p} \leq 1 .
$$


More conditions on $a(t)$ will be presented later. It is clear that a fixed point of the operator $S$ in $C_{a}$ is a constant-sign solution of (1.1) in $\left(L^{p}[0, T]\right)^{n}$.

Let $0<\beta<\alpha$. Define

$$
\Omega_{\alpha}=\left\{u \in B \mid\|u\|_{p}<\alpha\right\} \quad \text { and } \quad \Omega_{\beta}=\left\{u \in B \mid\|u\|_{p}<\beta\right\} .
$$

Our next lemma shows that $S$ maps $C_{a} \cap\left(\bar{\Omega}_{\alpha} \backslash \Omega_{\beta}\right)$ into $C_{a}$.

Lemma 3.3. Let $(\mathrm{C} 1)-(\mathrm{C} 4)$ hold. Assume

(C7) for each $1 \leq i \leq n$,

$$
\theta_{i} f_{i}(t, u)>0, \text { a.e. } t \in[0, T], \quad \text { all } u \in \hat{K}
$$

where

$$
\hat{K}=\left\{u \in B \mid \text { for each } 1 \leq i \leq n, \theta_{i} u_{i}(t)>0 \text { for a.e. } t \in[0, T]\right\} ;
$$

moreover, $\theta_{i} f_{i}$ is 'nondecreasing' in the sense that if $x \leq \theta_{j} u_{j} \leq y$ for some $j \in\{1,2, \cdots, n\}$, then for a.e. $t \in[0, T]$,

$$
\begin{aligned}
\theta_{i} f_{i}\left(t, u_{1}, \cdots, \theta_{j} x, \cdots, u_{n}\right) & \leq \theta_{i} f_{i}\left(t, u_{1}, \cdots, u_{j}, \cdots, u_{n}\right) \\
& \leq \theta_{i} f_{i}\left(t, u_{1}, \cdots, \theta_{j} y, \cdots, u_{n}\right)
\end{aligned}
$$

(C8) there exists a function $a \in L^{p}[0, T]$ with $a(t)>0$ for a.e. $t \in$ $[0, T]$ and $\|a\|_{p} \leq 1$ such that the following holds for each $1 \leq i \leq n$ and any $R>0$,

$$
\begin{gathered}
\int_{0}^{t} g_{i}(t, s) \theta_{i} f_{i}\left(s, \theta_{1} R a(s), \theta_{2} R a(s), \cdots, \theta_{n} R a(s)\right) d s \\
\geq a(t) \cdot \max _{1 \leq j \leq n} M_{j} \psi_{j}(R), \text { a.e. } t \in[0, T]
\end{gathered}
$$

where $\psi_{j}$ is defined in (C6) (see Remark 3.1 for alternative definition of $\psi_{j}$ ).

Then, the operator $S$ maps $C_{a} \cap\left(\bar{\Omega}_{\alpha} \backslash \Omega_{\beta}\right)$ into $C_{a}$. 
Proof. Let $u \in C_{a} \cap\left(\bar{\Omega}_{\alpha} \backslash \Omega_{\beta}\right)$. From Lemma 3.1, we already have $S u \in B$. Moreover, in view of (C3) and (C7), it follows that

$$
\begin{gathered}
\theta_{i}\left(S_{i} u\right)(t)=\int_{0}^{t} g_{i}(t, s) \theta_{i} f_{i}(s, u(s)) d s \geq 0, \\
\text { a.e. } t \in[0, T], \quad 1 \leq i \leq n .
\end{gathered}
$$

We shall show that $\theta_{i}\left(S_{i} u\right)(t) \geq a(t)\|u\|_{p}$ for a.e. $t \in[0, T]$ and each $1 \leq i \leq n$. Since $u \in C_{a} \cap\left(\bar{\Omega}_{\alpha} \backslash \Omega_{\beta}\right)$, there exists $R \in[\beta, \alpha]$ such that

$$
\begin{gathered}
\|u\|_{p}=R \quad \text { and } \quad \theta_{i} u_{i}(t) \geq a(t) R>0, \\
\text { a.e. } t \in[0, T], \quad 1 \leq i \leq n .
\end{gathered}
$$

and (C7), it is clear that for a.e. $t \in[0, T]$ and $1 \leq i \leq n$,

$$
\begin{aligned}
& \theta_{i}\left(S_{i} u\right)(t) \\
& \quad \geq \int_{0}^{t} g_{i}(t, s) \theta_{i} f_{i}\left(s, \theta_{1} R a(s), \theta_{2} R a(s), \cdots, \theta_{n} R a(s)\right) d s .
\end{aligned}
$$

On the other hand, using Hölder's inequality we have (3.7) which provides

$$
\left|S_{i} u\right|_{p} \leq M_{i}\left[\left|b_{i}\right|_{p_{2}}+\sum_{j=1}^{n} c_{i j}\left(\left|u_{j}\right|_{p}\right)^{\frac{p}{p_{2}}}\right] \leq M_{i} \psi_{i}\left(\|u\|_{p}\right)=M_{i} \psi_{i}(R),
$$

(note that $\psi_{i}$ is defined in $(\mathrm{C} 6)$ ). It follows that

$$
\|S u\|_{p} \leq \max _{1 \leq j \leq n} M_{j} \psi_{j}(R)
$$

Now, using (3.28) in (3.27) yields

$$
\begin{aligned}
\theta_{i}\left(S_{i} u\right)(t) & \geq \frac{\int_{0}^{t} g_{i}(t, s) \theta_{i} f_{i}\left(s, \theta_{1} R a(s), \theta_{2} R a(s), \cdots, \theta_{n} R a(s)\right) d s}{\max _{1 \leq j \leq n} M_{j} \psi_{j}(R)}\|S u\|_{p} \\
& \geq a(t)\|S u\|_{p}, \quad \text { a.e. } t \in[0, T], \quad 1 \leq i \leq n
\end{aligned}
$$


where the last inequality follows from (C8). This completes the proof.

$\square$

We are now ready to apply Theorem 2.2 to get the existence of a constant-sign solution in $\left(L^{p}[0,1]\right)^{n}$.

Theorem 3.3. Let $(\mathrm{C} 1)-(\mathrm{C} 4),(\mathrm{C} 7)$ and $(\mathrm{C} 8)$ hold. Assume

(C9) there exists $\alpha>0$ such that for each $1 \leq i \leq n$,

$$
M_{i} \psi_{i}(\alpha) \leq \alpha
$$

where $\psi_{i}$ is defined in $(\mathrm{C} 6)$;

(C10) there exists $\beta(\neq \alpha)>0$ such that for each $1 \leq i \leq n$,

$$
\int_{0}^{T}\left[\int_{0}^{t} g_{i}(t, s) \theta_{i} f_{i}\left(s, \theta_{1} \beta a(s), \theta_{2} \beta a(s), \cdots, \theta_{n} \beta a(s)\right) d s\right]^{p} d t \geq \beta^{p} .
$$

Then, the system (1.1) has at least one constant-sign solution $u \in$ $\left(L^{p}[0, T]\right)^{n}$ such that

(a) $\alpha \leq\|u\|_{p} \leq \beta \quad$ and $\quad \theta_{i} u_{i}(t) \geq a(t) \alpha, \quad$ a.e. $t \in[0, T], 1 \leq i \leq n$ if $\alpha<\beta$;

(b) $\beta \leq\|u\|_{p} \leq \alpha$ and $\theta_{i} u_{i}(t) \geq a(t) \beta, \quad$ a.e. $t \in[0, T], 1 \leq i \leq n$ if $\beta<\alpha$.

Proof. Without any loss of generality, let $\beta<\alpha$. Since $S: C_{a} \cap$ $\left(\bar{\Omega}_{\alpha} \backslash \Omega_{\beta}\right) \rightarrow C_{a}$ is continuous and completely continuous (Lemmas 3.13.3), it suffices to show that (i) $\|S u\|_{p} \leq\|u\|_{p}$ for $u \in C_{a} \cap \partial \Omega_{\alpha}$, and (ii) $\|S u\|_{p} \geq\|u\|_{p}$ for $u \in C_{a} \cap \partial \Omega_{\beta}$.

To verify (i), let $u \in C_{a} \cap \partial \Omega_{\alpha}$. Then,

$$
\|u\|_{p}=\alpha \text { and } \theta_{i} u_{i}(t) \geq a(t) \alpha>0, \quad \text { a.e. } t \in[0, T], \quad 1 \leq i \leq n .
$$

From the proof of Lemma 3.3, we obtain (3.28) $\left.\right|_{R=\alpha}$ and hence noting (C9) we find

$$
\|S u\|_{p} \leq \max _{1 \leq j \leq n} M_{j} \psi_{j}(\alpha) \leq \alpha=\|u\|_{p} .
$$

Next, to prove (ii), let $u \in C_{a} \cap \partial \Omega_{\beta}$. So 


$$
\|u\|_{p}=\beta \text { and } \theta_{i} u_{i}(t) \geq a(t) \beta>0, \quad \text { a.e. } t \in[0, T], \quad 1 \leq i \leq n .
$$

Now $\|S u\|_{p}=\left|S_{i} u\right|_{p}$ for some $i \in\{1,2, \cdots, n\}$. Thus, using (3.25), $\left.(3.27)\right|_{R=\beta}$ and (C10) gives

$$
\begin{aligned}
& \|S u\|_{p}=\left|S_{i} u\right|_{p}=\left\{\int_{0}^{T}\left[\theta_{i}\left(S_{i} u\right)(t)\right]^{p} d t\right\}^{\frac{1}{p}} \\
& \geq\left\{\int_{0}^{T}\left[\int_{0}^{t} g_{i}(t, s) \theta_{i} f_{i}\left(s, \theta_{1} \beta a(s), \theta_{2} \beta a(s), \cdots, \theta_{n} \beta a(s)\right) d s\right]^{p} d t\right\}^{\frac{1}{p}} \\
& \geq \beta=\|u\|_{p} .
\end{aligned}
$$

Having obtained (i) and (ii), it follows from Theorem 2.2 that $S$ has a fixed point $u \in C_{a} \cap\left(\bar{\Omega}_{\alpha} \backslash \Omega_{\beta}\right)$. Therefore, conclusion (b) follows immediately.

Remark 3.3. In (C9) if we have strict inequality instead, i.e., condition (C6), then from the proof of Theorem 3.3 we see that a fixed point $u$ of $S$ must satisfy $\|u\|_{p} \neq \alpha$. Similarly, if the inequality in (C10) is strict, i.e.,

$$
\int_{0}^{T}\left[\int_{0}^{t} g_{i}(t, s) \theta_{i} f_{i}\left(s, \theta_{1} \beta a(s), \theta_{2} \beta a(s), \cdots, \theta_{n} \beta a(s)\right) d s\right]^{p} d t>\beta^{p},
$$

then a fixed point $u$ of $S$ must fulfill $\|u\|_{p} \neq \beta$. Hence, with strict inequalities in (C9) and (C10), the conclusion of Theorem 3.3 becomes: the system (1.1) has at least one constant-sign solution $u \in\left(L^{p}[0, T]\right)^{n}$ such that

(a) $\alpha<\|u\|_{p}<\beta$ and $\theta_{i} u_{i}(t)>a(t) \alpha$, a.e.t $\in[0, T], \quad 1 \leq i \leq n$ if $\alpha<\beta$;

(b) $\beta<\|u\|_{p}<\alpha$ and $\theta_{i} u_{i}(t)>a(t) \beta, \quad$ a.e. $t \in[0, T], \quad 1 \leq i \leq n$ if $\beta<\alpha$.

Our next result gives the existence of multiple constant-sign solutions of $(1.1)$ in $\left(L^{p}[0, T]\right)^{n}$. 
Theorem 3.4. Assume $(\mathrm{C} 1)-(\mathrm{C} 5),(\mathrm{C} 7)$ and $(\mathrm{C} 8)$ hold. Let $(\mathrm{C} 6)$ be satisfied for $\alpha=\alpha_{0},(\mathrm{C} 9)$ be satisfied for $\alpha=\alpha_{\ell}, \ell=1,2, \cdots, k$, and (C10) be satisfied for $\beta=\beta_{\ell}, \ell=1,2, \cdots, m$.

(a) If $m=k+1$ and $0<\beta_{1}<\alpha_{1}<\cdots<\beta_{k}<\alpha_{k}<\beta_{k+1}$, then (1.1) has (at least) $2 k$ constant-sign solutions $u^{1}, \cdots, u^{2 k} \in\left(L^{p}[0, T]\right)^{n}$ such that

$$
\beta_{1} \leq\left\|u^{1}\right\|_{p} \leq \alpha_{1} \leq\left\|u^{2}\right\|_{p} \leq \beta_{2} \leq \cdots \leq \alpha_{k} \leq\left\|u^{2 k}\right\|_{p} \leq \beta_{k+1} .
$$

(b) If $m=k$ and $0<\beta_{1}<\alpha_{1}<\cdots<\beta_{k}<\alpha_{k}$, then (1.1) has (at least) $2 k-1$ constant-sign solutions $u^{1}, \cdots, u^{2 k-1} \in\left(L^{p}[0, T]\right)^{n}$ such that

$$
\beta_{1} \leq\left\|u^{1}\right\|_{p} \leq \alpha_{1} \leq\left\|u^{2}\right\|_{p} \leq \beta_{2} \leq \cdots \leq \beta_{k} \leq\left\|u^{2 k-1}\right\|_{p} \leq \alpha_{k} .
$$

(c) If $m=k+1$ and $0<\alpha_{0}<\beta_{1}<\alpha_{1}<\cdots<\alpha_{m-1}<\beta_{m}$, then (1.1) has (at least) $2 m$ constant-sign solutions $u^{0}, \cdots, u^{2 m-1} \in\left(L^{p}[0, T]\right)^{n}$ such that

$$
\begin{aligned}
0 \leq\left\|u^{0}\right\|_{p}<\alpha_{0}<\left\|u^{1}\right\|_{p} \leq \beta_{1} \leq\left\|u^{2}\right\|_{p} & \leq \alpha_{1} \leq \cdots \\
& \leq \alpha_{m-1} \leq\left\|u^{2 m-1}\right\|_{p} \leq \beta_{m} .
\end{aligned}
$$

(d) If $m=k$ and $0<\alpha_{0}<\beta_{1}<\alpha_{1}<\cdots<\beta_{m}<\alpha_{m}$, then (1.1) has (at least) $2 m+1$ constant-sign solutions $u^{0}, \cdots, u^{2 m} \in\left(L^{p}[0, T]\right)^{n}$ such that

$$
\begin{aligned}
0 \leq\left\|u^{0}\right\|_{p}<\alpha_{0}<\left\|u^{1}\right\|_{p} \leq \beta_{1} \leq\left\|u^{2}\right\|_{p} \leq \alpha_{1} & \leq \cdots \\
& \leq \beta_{m} \leq\left\|u^{2 m}\right\|_{p} \leq \alpha_{m} .
\end{aligned}
$$

Proof. In (a) and (b), we just apply Theorem 3.3 repeatedly. In (c) and (d), Theorem 3.2 is used to obtain the existence of $u^{0} \in\left(L^{p}[0, T]\right)^{n}$ with $0 \leq\left\|u^{0}\right\|_{p}<\alpha_{0}$, the results then follow by repeated use of Theorem 3.3.

Remark 3.4. Suppose in Theorem 3.4 we have some strict inequalities in (C9) and (C10), say, involving $\alpha_{i}$ and $\beta_{j}$ for some $i \in\{1,2, \cdots, k\}$ 
and some $j \in\{1,2, \cdots, m\}$. Then, noting Remark 3.3, those inequalities in the conclusion involving $\alpha_{i}$ and $\beta_{j}$ will also be strict.

We shall now illustrate two applications of Theorem 3.3.

Example 3.1. In nonlinear diffusion and percolation problems (see $[8,9]$ and the references cited therein), the system (1.1) arises where $g_{i}$ is a convolution kernel, i.e.,

$u_{i}(t)=\int_{0}^{t} g_{i}(t-s) f_{i}\left(s, u_{1}(s), u_{2}(s), \cdots, u_{n}(s)\right) d s$, a.e. $t \in[0, T], 1 \leq i \leq n$.

In particular, Bushell and Okrasiński [8] investigated a special case of the above system given by

$$
y(t)=\int_{0}^{t}(t-s)^{\gamma-1} f(y(s)) d s, \text { a.e. } t \in[0, T]
$$

where $\gamma>1$. To generalize their problem and also to illustrate the usefulness of the results obtained for (1.1), we shall consider the system

$$
\begin{gathered}
u_{i}(t)=\int_{0}^{t}(t-s)^{\gamma_{i}-1} f\left(s, u_{1}(s), u_{2}(s), \cdots, u_{n}(s)\right) d s \\
\text { a.e. } t \in[0, T], 1 \leq i \leq n
\end{gathered}
$$

where

$$
n=2, \quad T=1, \quad \gamma_{1}=2, \quad \gamma_{2}=3 \quad \text { and } \quad f(t, u)=u_{1}^{\frac{1}{4}}+u_{2}^{\frac{1}{4}}
$$

Suppose we are interested in seeking positive solutions of (3.29), (3.30) in $\left(L^{2}[0,1]\right)^{2}$. So we fix $\theta_{i}=1,1 \leq i \leq n$ and $p=2$. Choose $p_{1}=p_{2}=2$.

Clearly, conditions (C1), (C3) and (C7) are satisfied. Condition (C2) also holds with $b_{i}=0$ and $c_{i j}=1$. In (C4), we compute that

$$
\begin{aligned}
M_{1} & =\left[\int_{0}^{1}\left(\int_{s}^{1}(t-s)^{2} d t\right) d s\right]^{\frac{1}{2}}=\sqrt{\frac{1}{12}} \\
\text { and } \quad M_{2} & =\left[\int_{0}^{1}\left(\int_{s}^{1}(t-s)^{4} d t\right) d s\right]^{\frac{1}{2}}=\sqrt{\frac{1}{30}} .
\end{aligned}
$$


Further, using Hölder's inequality, we obtain

$$
\begin{aligned}
\left\{\int_{0}^{1}[f(t, u(t))]^{p_{2}} d t\right\}^{\frac{1}{p_{2}}} & =\left\{\int_{0}^{1}\left[\left(u_{1}(t)\right)^{\frac{1}{4}}+\left(u_{2}(t)\right)^{\frac{1}{4}}\right]^{2} d t\right\}^{\frac{1}{2}} \\
& \leq\left\{\int_{0}^{1} 2\left[\left(u_{1}(t)\right)^{\frac{1}{2}}+\left(u_{2}(t)\right)^{\frac{1}{2}}\right] d s\right\}^{\frac{1}{2}} \\
& \leq 2^{\frac{1}{2}}\left\{\left[\int_{0}^{1}\left(u_{1}(t)\right)^{2} d t\right]^{\frac{1}{4}}+\left[\int_{0}^{1}\left(u_{2}(t)\right)^{2} d t\right]^{\frac{1}{4}}\right\}^{\frac{1}{2}} \\
& =2^{\frac{1}{2}}\left\{\left|u_{1}\right|_{2}^{\frac{1}{2}}+\left|u_{2}\right|_{2}^{\frac{1}{2}}\right\}^{\frac{1}{2}} \\
& \leq 2^{\frac{1}{2}}\left\{2\|u\|_{2}^{\frac{1}{2}}\right\}^{\frac{1}{2}} \\
& =2\|u\|_{2}^{\frac{1}{4}} \equiv \psi\left(\|u\|_{2}\right)
\end{aligned}
$$

where the function $\psi$ is defined by

$$
\psi(x)=2 x^{\frac{1}{4}} .
$$

In view of Remark 3.1, we shall use the $\psi$ defined in (3.31) in conditions (C8) and (C9).

Now, the inequality in $(\mathrm{C} 8)$ reduces to

$$
\begin{gathered}
\int_{0}^{t} g_{i}(t, s) 2(R a(s))^{\frac{1}{4}} d s \geq a(t) \cdot \max _{j \in\{1,2\}} M_{j} \psi(R)=a(t) \cdot M_{1}\left(2 R^{\frac{1}{4}}\right), \\
\text { a.e. } t \in[0,1], \quad i=1,2
\end{gathered}
$$

or

$$
\int_{0}^{t} g_{i}(t, s)(a(s))^{\frac{1}{4}} d s \geq a(t) \cdot M_{1}, \quad \text { a.e. } t \in[0,1], i=1,2
$$

Let 


$$
a(t)=\left(\frac{1}{12}\right)^{\frac{2}{3}} t^{4} .
$$

It is clear that $a(t)>0$ for a.e. $t \in[0,1]$ and $\|a\|_{2}=0.063595 \leq 1$. We shall check that $a(t)$ fulfills (3.32). Indeed, by direct integration $\left.(3.32)\right|_{i=1}\left(g_{1}(t, s)=t-s\right)$ and $\left.(3.32)\right|_{i=2}\left(g_{2}(t, s)=(t-s)^{2}\right)$ respectively reduce to

$$
2 \geq t, \quad \text { a.e. } t \in[0,1]
$$

and

$$
t^{4} \geq t^{4}, \quad \text { a.e. } t \in[0,1]
$$

which are trivially true. Hence, we have shown that (C8) is satisfied with $a(t)$ defined in (3.33).

Next, the condition $(\mathrm{C} 9)$ reduces to

$$
M_{i} \psi(\alpha) \leq \alpha, \quad i=1,2
$$

or equivalently $2 M_{1} \leq \alpha^{\frac{3}{4}}$, which yields $\alpha \geq 0.48075$. Finally, (C10) leads to

$$
\int_{0}^{1}\left[\int_{0}^{t} g_{i}(t, s) 2(\beta a(s))^{\frac{1}{4}} d s\right]^{2} d t \geq \beta^{2}, i=1,2
$$

which gives $\beta \leq 0.010461$. Hence, (C9) and (C10) are satisfied if

$$
\alpha \geq 0.48075 \quad \text { and } \quad \beta \leq 0.010461 \text {. }
$$

Since $(\mathrm{C} 1)-(\mathrm{C} 4)$ and $(\mathrm{C} 7)-(\mathrm{C} 10)$ are satisfied, Theorem 3.3 is applicable and we conclude that (3.29), (3.30) has at least one positive solution $u \in\left(L^{2}[0,1]\right)^{2}$ with

$$
\begin{gathered}
\beta \leq\|u\|_{2} \leq \alpha \quad \text { and } \quad u_{i}(t) \geq\left[\left(\frac{1}{12}\right)^{\frac{2}{3}} t^{4}\right] \beta \\
\text { a.e. } t \in[0,1], \quad i=1,2
\end{gathered}
$$

Noting the ranges in (3.34), it follows from (3.35) that 


$$
\begin{aligned}
0.010461 & \leq\|u\|_{2} \leq 0.48075 \text { and } \\
u_{i}(t) & \geq\left[\left(\frac{1}{12}\right)^{\frac{2}{3}} t^{4}\right](0.010461), \quad \text { a.e. } t \in[0,1], i=1,2 .
\end{aligned}
$$

Example 3.2. Consider the system

$$
\begin{cases}u_{1}(t)=\int_{0}^{t} s t^{-\frac{3}{2}}\left[\left(u_{1}(s)\right)^{\frac{1}{4}}+\left(u_{2}(s)\right)^{\frac{1}{4}}\right] d s, & \text { a.e. } t \in[0,1] \\ u_{2}(t)=\int_{0}^{t} s^{2} t^{-\frac{3}{2}}\left[\left(u_{1}(s)\right)^{\frac{1}{4}}+\left(u_{2}(s)\right)^{\frac{1}{4}}\right] d s, & \text { a.e. } t \in[0,1] .\end{cases}
$$

Here,

$$
\begin{gathered}
n=2, \quad T=1, \quad g_{1}(t, s)=s t^{-\frac{3}{2}} \\
g_{2}(t, s)=s^{2} t^{-\frac{3}{2}} \quad \text { and } \quad f(t, u)=u_{1}^{\frac{1}{4}}+u_{2}^{\frac{1}{4}} .
\end{gathered}
$$

Suppose we are interested in seeking positive solutions of (3.36) in $\left(L^{2}[0,1]\right)^{2}$. Thus, we fix $\theta_{i}=1,1 \leq i \leq n$ and $p=p_{1}=p_{2}=2$.

Clearly, conditions $(\mathrm{C} 1),(\mathrm{C} 3)$ and $(\mathrm{C} 7)$ are satisfied. Condition $(\mathrm{C} 2)$ also holds with $b_{i}=0$ and $c_{i j}=1$. Condition (C4) is satisfied with $M_{1}=\sqrt{\frac{1}{3}}$ and $M_{2}=\sqrt{\frac{1}{15}}$.

From Example 3.1, we shall use the $\psi$ defined in (3.31) in conditions (C8) and (C9).

As before, we see that the inequality in (C8) reduces to (3.32). Let

$$
a(t)=\left(\frac{3}{16}\right)^{\frac{2}{3}} t^{4}
$$

It is clear that $a(t)>0$ for a.e. $t \in[0,1]$ and $\|a\|_{2}=0.10920 \leq 1$. We shall check that $a(t)$ fulfills (3.32). Indeed, by direct integration $\left.(3.32)\right|_{i=1}$ and (3.32) $\left.\right|_{i=2}$ respectively reduce to 


$$
\frac{4}{3} \geq t^{\frac{5}{2}}, \quad \text { a.e. } t \in[0,1]
$$

and

$$
1 \geq t^{\frac{3}{2}}, \quad \text { a.e. } t \in[0,1]
$$

which are trivially true. Hence, we have shown that (C8) is satisfied with $a(t)$ defined in (3.37).

Finally, by direct computation we see that (C9) and (C10) are satisfied if

$$
\alpha \geq 1.2114 \quad \text { and } \quad \beta \leq 0.082852 .
$$

Now that $(\mathrm{C} 1)-(\mathrm{C} 4)$ and $(\mathrm{C} 7)-(\mathrm{C} 10)$ are satisfied, we can apply Theorem 3.3 to conclude that (3.36) has at least one positive solution $u \in\left(L^{2}[0,1]\right)^{2}$ with

$$
\begin{gathered}
\beta \leq\|u\|_{2} \leq \alpha \text { and } u_{i}(t) \geq\left[\left(\frac{3}{16}\right)^{\frac{2}{3}} t^{4}\right] \beta, \\
\text { a.e. } t \in[0,1], \quad i=1,2 .
\end{gathered}
$$

Noting the ranges in (3.38), it follows from (3.39) that

$$
\begin{array}{r}
0.082852 \leq\|u\|_{2} \leq 1.2114 \text { and } u_{i}(t) \geq\left[\left(\frac{3}{16}\right)^{\frac{2}{3}} t^{4}\right](0.082852), \\
\text { a.e. } t \in[0,1], i=1,2 .
\end{array}
$$

4. Existence in Orlicz Space We shall consider the system (1.1) where, for each $1 \leq i \leq n, g_{i}:[0, T] \times[0, t] \rightarrow \mathbb{R}^{N \times N}$ is a matrix valued kernel function and $f_{i}:[0, T] \times\left(\mathbb{R}^{N}\right)^{n} \rightarrow \mathbb{R}^{N}$ is a single-valued nonlinear function. We shall establish the existence of a constant-sign solution $u \in\left(L_{\phi}\right)^{n}$ of (1.1) where $L_{\phi}$ is an Orlicz space. Throughout this section, let $\theta_{i} \in\{1,-1\}, 1 \leq i \leq n$ be fixed. 
Let $x=\left(x_{1}, x_{2}, \cdots, x_{N}\right)^{T}$ and $y=\left(y_{1}, y_{2}, \cdots, y_{N}\right)^{T}$ be in $\mathbb{R}^{N}$. Throughout, by $x \geq y$ we shall mean $x_{i} \geq y_{i}$ for each $1 \leq i \leq N$. Similarly, if $x, y \in \mathbb{R}^{N \times N}$ (real $N \times N$ matrices), then $x \geq y$ also means inequality in the componentwise sense.

Let $B$ be a Banach space. Let the operator $S: B \rightarrow\left(\mathbb{R}^{N}\right)^{n}$ be defined by

$$
S u(t)=\left(S_{1} u(t), S_{2} u(t), \cdots, S_{n} u(t)\right), \quad \text { a.e. } t \in[0, T]
$$

where

$$
S_{i} u(t)=\int_{0}^{t} g_{i}(t, s) f_{i}(s, u(s)) d s, \quad \text { a.e. } t \in[0, T], \quad 1 \leq i \leq n .
$$

Clearly, a fixed point of the operator $S$ is a solution of the system (1.1). We observe that the operator $S_{i}$ can be written as

$$
S_{i}=A_{i} F_{i}
$$

where $F_{i}: B \rightarrow \mathbb{R}^{N}$ is defined by

$$
F_{i} u(t)=f_{i}(t, u(t)), \quad \text { a.e. } t \in[0, T]
$$

and $A_{i}: \mathbb{R}^{N} \rightarrow \mathbb{R}^{N}$ is given by

$$
A_{i} x(t)=\int_{0}^{t} g_{i}(t, s) x(s) d s, \quad \text { a.e. } t \in[0, T] .
$$

Our first result is a general existence principle in $B$. The main tool used is the Leray-Schauder alternative (Theorem 2.1).

Theorem 4.1. Let $X=\left(X,|\cdot|_{X}\right)$ be a Banach space and let $X^{n}=X \times X \times \cdots \times X$ ( $n$ times) be equipped with the norm $\|\cdot\|$ where

$$
\|u\|=\max _{1 \leq i \leq n}\left|u_{i}\right|_{X}, \quad u \in X^{n} .
$$

Let $Y$ be a Banach space. For each $1 \leq i \leq n$, suppose

$$
F_{i}: X^{n} \rightarrow Y \quad \text { and } \quad A_{i}: Y \rightarrow X
$$


and

$$
A_{i} F_{i}: X^{n} \rightarrow X \text { is continuous and completely continuous. }
$$

Moreover, assume there is a positive constant $M_{0}$, independent of $\lambda$, with

$$
\|u\| \neq M_{0}
$$

for any solution $u \in X^{n}$ of the system

$$
u_{i}=\lambda A_{i} F_{i} u, \quad \text { a.e. }
$$

where $1 \leq i \leq n$ and $0<\lambda<1$. Then, the system (1.1) has a solution $u^{*} \in X^{n}$ with $\left\|u^{*}\right\| \leq M_{0}$.

Proof. Clearly, a solution of $(4.9)_{\lambda}$ is a fixed point of the equation $u=\lambda S u$ where $S$ is defined in (4.1), (4.2). Now (4.7) guarantees that $S$ is continuous and completely continuous. In the context of Theorem 2.1 , let

$$
U=\left\{u \in X^{n} \mid\|u\|<M_{0}\right\} .
$$

Since $\|u\| \neq M_{0}$, where $u$ is any solution of $(4.9)_{\lambda}$, we cannot have conclusion (b) of Theorem 2.1, hence conclusion (a) of Theorem 2.1 must hold, i.e., the system (1.1) has a solution $u^{*} \in \bar{U}$ with $\left\|u^{*}\right\| \leq M_{0}$.

We shall now tackle the existence of a solution $u$ of (1.1) with $u_{i} \in X, 1 \leq i \leq n$ where $X$ is an Orlicz space. To introduce Orlicz spaces, we require the following definition.

Definition 4.1. A function $P$ is called an $N$-function if it admits a representation

$$
P(u)=\int_{0}^{|u|} p(t) d t
$$


where the function $p$ is right continuous for $t \geq 0$, positive for $t>0$ and nondecreasing and satisfies the conditions

$$
p(0)=0, \quad \lim _{t \rightarrow \infty} p(t)=\infty .
$$

The functions $P_{1}(u)=\frac{|u|^{\alpha}}{\alpha}, \alpha>1$ and $P_{2}(u)=e^{|u|}-|u|-1$ are examples of $N$-functions.

Let $p$ be as above. Let $q(s)=\sup _{p(t) \leq s} t$. The functions

$$
P(u)=\int_{0}^{|u|} p(t) d t, \quad Q(v)=\int_{0}^{|v|} q(s) d s
$$

are called complementary $N$-functions. Note $N_{1}(v)=\frac{|v|^{\beta}}{\beta}, \frac{1}{\alpha}+\frac{1}{\beta}=$ 1 , is the $N$-function complementary to the $N$-function $P_{1}$ whereas $N_{2}(v)=(1+|v|) \ln (1+|v|)-|v|$ is the $N$-function complementary to the $N$-function $P_{2}$.

Now, let $P$ and $Q$ be complementary $N$-functions. The Orlicz class, denoted by $\mathcal{O}_{P}$, consists of measurable functions $y:[0, d] \rightarrow \mathbb{R}^{N}$ for which

$$
\rho(y ; P)=\int_{0}^{d} P(y(x)) d x<\infty .
$$

We shall denote by $L_{P}\left([0, d], \mathbb{R}^{N}\right)$ the Orlicz space of all measurable functions $y:[0, d] \rightarrow \mathbb{R}^{N}$ for which

$$
|y|_{P, d}=\sup _{\substack{\rho(y ; Q) \leq 1 \\ v \in \mathcal{O}_{Q}}}\left|\int_{0}^{d} y(x) \cdot v(x) d x\right|<\infty .
$$

It is known that $\left(L_{P}\left([0, d], \mathbb{R}^{N}\right),|\cdot|_{P, d}\right)$ is a Banach space [14]. Let $E_{P}\left([0, d], \mathbb{R}^{N}\right)$ be the closure in $L_{P}\left([0, d], \mathbb{R}^{N}\right)$ of the set of all bounded functions. Note that $E_{P} \subseteq L_{P} \subseteq \mathcal{O}_{P}$. We have $E_{P}=L_{P}=\mathcal{O}_{P}$ if $P$ satisfies the $\left(\triangle_{2}\right)$ condition, which is

$\left(\triangle_{2}\right)$ there exist $\omega, y_{0} \geq 0$ such that for $y \geq y_{0}$, we have $P(2 y) \leq$ $\omega P(y)$.

Our first existence result in Orlicz space employs Theorem 4.1.

Theorem 4.2. Let $P$ and $Q$ be complementary $N$-functions. Assume 
(H1) $\phi$ is an $N$-function, and $Q$ satisfies the $\left(\triangle_{2}\right)$ condition;

(H2) for each $1 \leq i \leq n, g_{i}(t, \cdot) \in E_{P}\left([0, T], \mathbb{R}^{N \times N}\right)$ for a.e. $t \in[0, T]$, and the function $t \mapsto\left|g_{i}(t, \cdot)\right|_{P, T}$ belongs to $E_{\phi}([0, T], \mathbb{R})$;

(H3) for each $1 \leq i \leq n, f_{i}:[0, T] \times\left(\mathbb{R}^{N}\right)^{n} \rightarrow \mathbb{R}^{N}$ is a Carathéodory function, i.e.,

(i) the map $t \mapsto f_{i}(t, u)$ is measurable for all $u \in\left(\mathbb{R}^{N}\right)^{n}$,

(ii) the map $u \mapsto f_{i}(t, u)$ is continuous for almost all $t \in[0, T]$;

(H4) for each $r>0$ and $1 \leq i \leq n$, there exists $\eta_{r, i} \in L_{Q}([0, T], \mathbb{R})$ and $K_{r, i} \geq 0$ such that

$$
\left|f_{i}(t, u)\right| \leq \eta_{r, i}(t)+K_{r, i} Q^{-1}\left(\phi\left(\frac{\left|u_{i}\right|}{r}\right)\right) \text {, a.e. } t \in[0, T] \text {, all } u \in\left(\mathbb{R}^{N}\right)^{n} .
$$

Moreover, assume there is a positive constant $M_{0}$, independent of $\lambda$, with

$$
\|u\|_{\phi, T}=\max _{1 \leq i \leq n}\left|u_{i}\right|_{\phi, T} \neq M_{0}
$$

for any solution $u$ of $(4.9)_{\lambda}$. Then, the system (1.1) has a solution $u^{*} \in\left(E_{\phi}\left([0, T], \mathbb{R}^{N}\right)\right)^{n}$ with $\left\|u^{*}\right\|_{\phi, T} \leq M_{0}$.

Proof. It follows immediately from Lemma 16.3 and Theorem 16.3 of [14] (take $M_{1}=Q, M_{2}=\phi$ and $\left.N_{1}=P\right)$ that $A_{i}: E_{Q}\left([0, T], \mathbb{R}^{N}\right) \rightarrow$ $E_{\phi}\left([0, T], \mathbb{R}^{N}\right)$ is continuous and completely continuous. Let

$$
U=\left\{u \in\left(E_{\phi}\left([0, T], \mathbb{R}^{N}\right)\right)^{n} \mid\|u\|_{\phi, T}<M_{0}\right\} .
$$

Applying Theorem 17.6 in [14] (with $M_{2}=Q$ and $M_{1}=\phi$ ), we deduce that $F_{i}: \bar{U} \rightarrow E_{Q}\left([0, T], \mathbb{R}^{N}\right)$ is continuous and $F_{i}$ maps bounded sets into bounded sets. Thus $A_{i} F_{i}: \bar{U} \rightarrow E_{\phi}\left([0, T], \mathbb{R}^{N}\right)$ is continuous ( $A_{i}$ is continuous and $F_{i}$ is also continuous) and completely continuous ( $A_{i}$ is completely continuous and $F_{i}$ maps bounded sets into bounded sets). With $X=E_{\phi}\left([0, T], \mathbb{R}^{N}\right)$ and $Y=E_{Q}\left([0, T], \mathbb{R}^{N}\right)$, the result now follows from Theorem 4.1.

Remark 4.1. By placing other conditions on $g_{i}$ and $f_{i}$ (see [14, Sections $15,16,17]$ ) we may deduce other existence results in an Orlicz space. 
Our next two results apply Theorem 4.2.

Theorem 4.3. Let $P$ and $Q$ be complementary $N$-functions. Let (H1)-(H4) hold. Assume

(H5) for each $1 \leq i \leq n, \hat{g}_{i}(t) \equiv\left|g_{i}(t, \cdot)\right|_{P, t}$ is bounded for $t \in[0, T]$;

(H6) for each $r>0$ and $1 \leq i \leq n$,

$$
\frac{1}{2} \int_{0}^{T}\left[1+\phi\left(b_{r, i}(t)\right)\right] d t<\int_{0}^{\infty}\left[1+\phi\left(\frac{2 \hat{g}_{i} K_{r, i}}{r} x\right)\right]^{-1} d x
$$

where

$$
\hat{g}_{i}=\sup _{t \in[0, T]} \hat{g}_{i}(t) \quad \text { and } \quad b_{r, i}(t)=\frac{2 \hat{g}_{i}(t)}{r}\left(\left|\eta_{r, i}\right|_{Q, t}+K_{r, i}\right) ;
$$

(H7) for each $1 \leq i \leq n$,

$$
\sup _{r \in(0, \infty)\left|q_{i}\right|_{\phi, T}\left\{\left|\eta_{r, i}\right|_{Q, T}+K_{r, i}+K_{r, i} I_{r, i}^{-1}\left(\frac{1}{2} \int_{0}^{T}\left[1+\phi\left(b_{r, i}(t)\right)\right] d t\right)\right\}}
$$

where

$q_{i}(t)=\left|g_{i}(t, \cdot)\right|_{P, T} \quad$ and $\quad I_{r, i}(z)=\int_{0}^{z}\left[1+\phi\left(\frac{2 \hat{g}_{i} K_{r, i}}{r} x\right)\right]^{-1} d x$

Then, the system $(1.1)$ has a solution $u \in\left(E_{\phi}\left([0, T], \mathbb{R}^{N}\right)\right)^{n}$.

Proof. In view of (H7), for any $1 \leq i \leq n$ there exists a positive constant $M_{0}$ such that

$$
\frac{M_{0}}{\left.\frac{\left|q_{i}\right|_{\phi, T}\left\{\left|\eta_{M_{0}, i}\right|_{Q, T}+K_{M_{0}, i}+\right.}{} K_{M_{0}, i} I_{M_{0}, i}^{-1}\left(\frac{1}{2} \int_{0}^{T}\left[1+\phi\left(b_{M_{0}, i}(t)\right)\right] d t\right)\right\}}
$$

For each $1 \leq i \leq n$, define $A_{i}: E_{Q}\left([0, T], \mathbb{R}^{N}\right) \rightarrow E_{\phi}\left([0, T], \mathbb{R}^{N}\right)$ by $(4.5)$, and $F_{i}:\left(E_{\phi}\left([0, T], \mathbb{R}^{N}\right)\right)^{n} \rightarrow E_{Q}\left([0, T], \mathbb{R}^{N}\right)$ by $(4.4)$. Let $u$ be 
a solution of $(4.9)_{\lambda}$ for some $\lambda \in(0,1)$ with $\|u\|_{\phi, T}=M_{0}$. Then, there exists some $j \in\{1,2, \cdots, n\}$ such that $\left|u_{j}\right|_{\phi, T}=M_{0}$.

By Lemma 16.3(a) of [14] (with $M_{2}=\phi, N_{1}=P, M_{1}=Q$ ), we have

$$
\left|A_{i} v\right|_{\phi, T} \leq\left|q_{i}\right|_{\phi, T} \cdot|v|_{Q, T}, \quad 1 \leq i \leq n
$$

Moreover, using [14, Theorem 10.5 with $k=1]$ gives

$$
\left|Q^{-1}\left(\phi\left(\frac{\left|u_{i}\right|}{M_{0}}\right)\right)\right|_{Q, t} \leq 1+\int_{0}^{t} \phi\left(\frac{\left|u_{i}(s)\right|}{M_{0}}\right) d s, \quad 1 \leq i \leq n
$$

Now applying (H4) $\left.\right|_{r=M_{0}}$, (4.13) and (4.14), we find

$$
\begin{aligned}
\left|u_{j}\right|_{\phi, T} & \leq\left|\int_{0}^{t} g_{j}(t, s) f_{j}(s, u(s)) d s\right|_{\phi, T} \\
(4.15) & \leq\left|\int_{0}^{t} g_{j}(t, s)\left\{\eta_{M_{0}, j}(s)+K_{M_{0}, j} Q^{-1}\left(\phi\left(\frac{\left|u_{j}\right|}{M_{0}}\right)\right)\right\} d s\right|_{\phi, T} \\
& \leq\left|q_{j}\right|_{\phi, T}\left\{\left|\eta_{M_{0}, j}\right|_{Q, T}+K_{M_{0}, j}\left|Q^{-1}\left(\phi\left(\frac{\left|u_{j}\right|}{M_{0}}\right)\right)\right|_{Q, T}\right\} \\
& \leq\left|q_{j}\right|_{\phi, T}\left\{\left|\eta_{M_{0}, j}\right|_{Q, T}+K_{M_{0}, j}\left[1+\int_{0}^{T} \phi\left(\frac{\left|u_{j}(s)\right|}{M_{0}}\right) d s\right]\right\} .
\end{aligned}
$$

On the other hand, using (H4), Hölder's inequality and (4.14), it follows from $(4.9)_{\lambda}$ that for a.e. $t \in[0, T]$,

$$
\begin{aligned}
\left|u_{j}(t)\right| & \leq\left|\int_{0}^{t} g_{j}(t, s) f_{j}(s, u(s)) d s\right| \\
(4.16) & \leq \hat{g}_{j}(t)\left\{\left|\eta_{M_{0}, j}\right|_{Q, t}+K_{M_{0}, j}\left|Q^{-1}\left(\phi\left(\frac{\left|u_{j}\right|}{M_{0}}\right)\right)\right|_{Q, t}\right\} \\
& \leq \hat{g}_{j}(t)\left(\left|\eta_{M_{0}, j}\right|_{Q, t}+K_{M_{0}, j}\right)+\hat{g}_{j} K_{M_{0}, j} \int_{0}^{t} \phi\left(\frac{\left|u_{j}(s)\right|}{M_{0}}\right) d s .
\end{aligned}
$$


Noting that $\phi(x+y) \leq \frac{1}{2}[\phi(2 x)+\phi(2 y)]$ for $x, y \geq 0$, from (4.16) we obtain for a.e. $t \in[0, T]$,

$$
\phi\left(\frac{\left|u_{j}(t)\right|}{M_{0}}\right) \leq \frac{1}{2} \phi\left(b_{M_{0}, j}(t)\right)+\frac{1}{2} \phi\left(\frac{2 \hat{g}_{j} K_{M_{0}, j}}{M_{0}} \int_{0}^{t} \phi\left(\frac{\left|u_{j}(s)\right|}{M_{0}}\right) d s\right) \text {. }
$$

Let

$$
w(t)=\int_{0}^{t} \phi\left(\frac{\left|u_{j}(s)\right|}{M_{0}}\right) d s .
$$

Then, (4.17) gives

$$
\begin{aligned}
2 w^{\prime}(t) & \leq \phi\left(b_{M_{0}, j}(t)\right)+\phi\left(\frac{2 \hat{g}_{j} K_{M_{0}, j}}{M_{0}} w(t)\right) \\
& \leq\left[1+\phi\left(b_{M_{0}, j}(t)\right)\right] \cdot\left[1+\phi\left(\frac{2 \hat{g}_{j} K_{M_{0}, j}}{M_{0}} w(t)\right)\right]
\end{aligned}
$$

or

$$
w^{\prime}(t)\left[1+\phi\left(\frac{2 \hat{g}_{j} K_{M_{0}, j}}{M_{0}} w(t)\right)\right]^{-1} \leq \frac{1}{2}\left[1+\phi\left(b_{M_{0}, j}(t)\right)\right]
$$

which, upon integrating from 0 to $T$, provides

$$
\begin{aligned}
\int_{0}^{w(T)}[1+\phi & \left.\left(\frac{2 \hat{g}_{j} K_{M_{0}, j}}{M_{0}} x\right)\right]^{-1} d x=I_{M_{0}, j}(w(T)) \\
& \leq \frac{1}{2} \int_{0}^{T}\left[1+\phi\left(b_{M_{0}, j}(t)\right)\right] d t<I_{M_{0}, j}(\infty) \text { (by (H6)). }
\end{aligned}
$$

Since $I_{M_{0}, j}$ is strictly increasing, it follows immediately from above that

(4.18) $w(T)=\int_{0}^{T} \phi\left(\frac{\left|u_{j}(s)\right|}{M_{0}}\right) d s \leq I_{M_{0}, j}^{-1}\left(\frac{1}{2} \int_{0}^{T}\left[1+\phi\left(b_{M_{0}, j}(t)\right)\right] d t\right)$.

Now, we substitute (4.18) into (4.15) to get

$$
\begin{aligned}
M_{0}= & \left|u_{j}\right|_{\phi, T} \leq\left|q_{j}\right|_{\phi, T}\left\{\left|\eta_{M_{0}, j}\right|_{Q, T}+K_{M_{0}, j}[1+w(T)]\right\} \\
\leq & \left|q_{j}\right|_{\phi, T}\left\{\left|\eta_{M_{0}, j}\right|_{Q, T}\right. \\
& \left.+K_{M_{0}, j}+K_{M_{0}, j} I_{M_{0}, j}^{-1}\left(\frac{1}{2} \int_{0}^{T}\left[1+\phi\left(b_{M_{0}, j}(t)\right)\right] d t\right)\right\} \\
< & M_{0} \text { (by (4.12)) }
\end{aligned}
$$


which is a contradiction. Hence, any solution $u$ of $(4.9)_{\lambda}$ must satisfy $\|u\|_{\phi, T} \neq M_{0}$. The condition (4.10) is satisfied and the conclusion is now immediate from Theorem 4.2.

Theorem 4.4. Let $P$ and $Q$ be complementary $N$-functions. Let (H1)-(H4) hold. Assume (H8) for each $1 \leq i \leq n$,

$$
\sup _{r \in(0, \infty)} \frac{r}{\left|q_{i}\right|_{\phi, T}\left(\left|\eta_{r, i}\right|_{Q, T}+2 K_{r, i}\right)}>1
$$

where $q_{i}(t)=\left|g_{i}(t, \cdot)\right|_{P, T}$. Then, the system (1.1) has a solution $u \in$ $\left(E_{\phi}\left([0, T], \mathbb{R}^{N}\right)\right)^{n}$.

Proof. In view of (H8), for any $1 \leq i \leq n$ there exists a positive constant $M_{0}$ such that

$$
\frac{M_{0}}{\left|q_{i}\right|_{\phi, T}\left(\left|\eta_{M_{0}, i}\right|_{Q, T}+2 K_{M_{0}, i}\right)}>1 .
$$

As in the proof of Theorem 4.3, let $u$ be a solution of $(4.9)_{\lambda}$ for some $\lambda \in(0,1)$ with $\|u\|_{\phi, T}=M_{0}$. Also, let $j \in\{1,2, \cdots, n\}$ be such that $\|u\|_{\phi, T}=\left|u_{j}\right|_{\phi, T}=M_{0}$.

Using a similar argument as before, we obtain (4.15). Now, we employ Lemma 9.2 in [14] to get

$$
\int_{0}^{T} \phi\left(\frac{\left|u_{j}(s)\right|}{M_{0}}\right) d s \leq \frac{\left|u_{j}\right|_{\phi, T}}{M_{0}}=\frac{M_{0}}{M_{0}}=1 .
$$

Substituting (4.20) into (4.15) immediately leads to

$$
M_{0}=\left|u_{j}\right|_{\phi, T} \leq\left|q_{j}\right|_{\phi, T}\left(\left|\eta_{M_{0}, j}\right|_{Q, T}+2 K_{M_{0}, j}\right)<M_{0} \text { (by (4.19)), }
$$

a contradiction.

Hence, any solution $u$ of $(4.9)_{\lambda}$ must satisfy $\|u\|_{\phi, T} \neq M_{0}$, i.e., we have the condition (4.10). The conclusion is now immediate from Theorem 4.2.

Remark 4.2. Let $p(>1)$ and $q$ be integers such that $\frac{1}{p}+\frac{1}{q}=1$. Consider the case $n=1$. In Theorem 3.2, the existence of a solution in $L^{p}[0, T]$ is established using the conditions (see (C2) and (C6)) 


$$
|f(t, u)| \leq \eta_{r}(t)+M_{r}|u|^{\frac{p}{q}}, \quad \text { a.e. } t \in[0, T], \text { all } u \in \mathbb{R}
$$

and

$$
\sup _{r \in(0, \infty)}\left(\frac{r}{a_{0}+a_{1} r^{\frac{p}{q}}}\right)>1
$$

where $a_{0}$ and $a_{1}$ are some fixed constants. We remark that our Theorems 4.3 and 4.4, which give the existence of a solution in an Orlicz space, are 'analogous' to Theorem 3.2 in the sense that if we let $\phi(x)=\frac{|x|^{p}}{p}$, then $Q(x)=\frac{|x|^{q}}{q}$, and so $Q^{-1}(x)=(q x)^{\frac{1}{q}}$ for $x \geq 0$. Then, (H4) with

$$
K_{r}=M_{r} \cdot r^{\frac{p}{q}}\left(\frac{p}{q}\right)^{\frac{1}{q}}
$$

reduces to (4.21), since with this $K_{r}$ we have

$$
K_{r} Q^{-1}\left(\phi\left(\frac{|u|}{r}\right)\right)=M_{r}|u|^{\frac{p}{q}} .
$$

Moreover, condition (H8) is 'parallel' to (4.22).

Remark 4.3. It is also possible to prove Theorem 4.4 using Schauder's fixed point theorem.

Remark 4.4. In Theorems 4.3 and 4.4, one could replace (H4) with the following condition:

(H4) there exists $\epsilon>0$ and for each $1 \leq i \leq n$, there exists $\eta_{M_{0}+\epsilon, i} \in L_{Q}([0, T], \mathbb{R})$ and $K_{M_{0}+\epsilon, i} \geq 0$ such that

$$
\begin{aligned}
\left|f_{i}(t, u)\right| \leq \eta_{M_{0}+\epsilon, i}(t)+K_{M_{0}+\epsilon, i} Q^{-1} & \left(\phi\left(\frac{\left|u_{i}\right|}{M_{0}+\epsilon}\right)\right), \\
& \text { a.e. } t \in[0, T], \text { all } u \in\left(\mathbb{R}^{N}\right)^{n}
\end{aligned}
$$

where $M_{0}$ is as in (4.12) (for Theorem 4.3) or as in (4.19) (for Theorem $4.4)$. 
Remark 4.5. The conditions (H7) and (H8) are respectively equivalent to

$(\mathrm{H} 7)^{\prime}$ there exists $\alpha>0$ such that for each $1 \leq i \leq n$,

$$
\left|q_{i}\right|_{\phi, T}\left\{\left|\eta_{\alpha, i}\right|_{Q, T}+K_{\alpha, i}+K_{\alpha, i} I_{\alpha, i}^{-1}\left(\frac{1}{2} \int_{0}^{T}\left[1+\phi\left(b_{\alpha, i}(t)\right)\right] d t\right)\right\}<\alpha
$$

where $q_{i}$ and $I_{\alpha, i}$ are defined in (H7);

$(\mathrm{H} 8)^{\prime}$ there exists $\alpha>0$ such that for each $1 \leq i \leq n$,

$$
\left|q_{i}\right|_{\phi, T}\left(\left|\eta_{\alpha, i}\right|_{Q, T}+2 K_{\alpha, i}\right)<\alpha .
$$

Moreover, with (H7) ${ }^{\prime}$ and $(\mathrm{H} 8)^{\prime}$ the conclusion of Theorems 4.3 and 4.4 becomes: the system (1.1) has a solution $u \in\left(E_{\phi}\left([0, T], \mathbb{R}^{N}\right)\right)^{n}$ such that $\|u\|_{\phi, T}<\alpha$.

Till now we have employed the Leray-Schauder alternative (Theorem $2.1)$ to obtain existence criteria in $\left(E_{\phi}\left([0, T], \mathbb{R}^{N}\right)\right)^{n}$. We shall next apply Krasnosel'skii's fixed point theorem (Theorem 2.2) to get further existence results for constant-sign solutions in $\left(E_{\phi}\left([0, T], \mathbb{R}^{N}\right)\right)^{n}$.

Consider the Banach space $\left(\left(E_{\phi}([0, T],)^{N}\right)^{n},\|\cdot\|_{\phi, T}\right)$. Define a cone in $\left(E_{\phi}\left([0, T], \mathbb{R}^{N}\right)\right)^{n}$ as

$$
\begin{array}{r}
C_{\gamma}=\left\{u \in\left(E_{\phi}\left([0, T], \mathbb{R}^{N}\right)\right)^{n} \mid \text { for each } 1 \leq i \leq n\right. \\
\left.\qquad \theta_{i} u_{i}(t) \geq \gamma(t)\|u\|_{\phi, T} \text { for a.e. } t \in[0, T]\right\}
\end{array}
$$

Here we assume

$\gamma(t)>0$, a.e. $t \in[0, T], \quad \gamma \in E_{\phi}\left([0, T], \mathbb{R}^{N}\right) \quad$ and $\quad|\gamma|_{\phi, T} \leq 1$.

More conditions on $\gamma(t)$ will be presented later. It is clear that a fixed point of the operator $S$ in $C_{\gamma}$ is a constant-sign solution of (1.1) in $\left(E_{\phi}\left([0, T], \mathbb{R}^{N}\right)\right)^{n}$. 
Let $0<\beta<\alpha$. Define

$$
\Omega_{\alpha}=\left\{u \in\left(E_{\phi}\left([0, T], \mathbb{R}^{N}\right)\right)^{n} \mid\|u\|_{\phi, T}<\alpha\right\}
$$

and

$$
\Omega_{\beta}=\left\{u \in\left(E_{\phi}\left([0, T], \mathbb{R}^{N}\right)\right)^{n} \mid\|u\|_{\phi, T}<\beta\right\} .
$$

Our next lemma shows that $S$ maps $C_{\gamma} \cap\left(\bar{\Omega}_{\alpha} \backslash \Omega_{\beta}\right)$ into $C_{\gamma}$.

Lemma 4.1. Let $P$ and $Q$ be complementary $N$-functions. Let (H1)(H4) hold. Assume

(H9) for each $1 \leq i \leq n, g_{i}(t, s):[0, T] \times[0, t] \rightarrow[0, \infty)^{N \times N}$ is such that the map $(t, s) \rightarrow g_{i}(t, s)$ is measurable, and $g_{i}(t, s)>0$, a.e. $t \in$ $[0, T]$, a.e. $s \in[0, t]$;

(H10) for each $1 \leq i \leq n$,

$$
\theta_{i} f_{i}(t, u)>0, \quad \text { a.e. } t \in[0, T], \text { all } u \in \hat{K}
$$

where

$$
\begin{gathered}
\hat{K}=\left\{u \in\left(E_{\phi}\left([0, T], \mathbb{R}^{N}\right)\right)^{n} \mid \text { for each } 1 \leq i \leq n,\right. \\
\left.\theta_{i} u_{i}(t)>0 \text { for } \quad \text { a.e. } t \in[0, T]\right\}
\end{gathered}
$$

moreover, $\theta_{i} f_{i}$ is 'nondecreasing' in the sense that if $x \leq \theta_{j} u_{j} \leq y$ for some $j \in\{1,2, \cdots, n\}$, then for a.e. $t \in[0, T]$,

$$
\begin{aligned}
\theta_{i} f_{i}\left(t, u_{1}, \cdots, \theta_{j} x, \cdots, u_{n}\right) & \leq \theta_{i} f_{i}\left(t, u_{1}, \cdots, u_{j}, \cdots, u_{n}\right) \\
& \leq \theta_{i} f_{i}\left(t, u_{1}, \cdots, \theta_{j} y, \cdots, u_{n}\right) ;
\end{aligned}
$$

(H11) there exists a function $\gamma \in E_{\phi}\left([0, T], \mathbb{R}^{N}\right)$ with $\gamma(t)>0$ for a.e. $t \in[0, T]$ and $|\gamma|_{\phi, T} \leq 1$ such that the following holds for each $1 \leq i \leq n$ and any $R>0$,

$$
\begin{aligned}
& \int_{0}^{t} g_{i}(t, s) \theta_{i} f_{i}\left(s, \theta_{1} R \gamma(s), \theta_{2} R \gamma(s), \cdots, \theta_{n} R \gamma(s)\right) d s \\
& \quad \geq \gamma(t) \cdot \max _{1 \leq j \leq n}\left|q_{j}\right|_{\phi, T}\left(\left|\eta_{R, j}\right|_{\phi, T}+2 K_{R, j}\right), \quad \text { a.e. } t \in[0, T] .
\end{aligned}
$$


Then, the operator $S$ maps $C_{\gamma} \cap\left(\bar{\Omega}_{\alpha} \backslash \Omega_{\beta}\right)$ into $C_{\gamma}$.

Proof. Let $u \in C_{\gamma} \cap\left(\bar{\Omega}_{\alpha} \backslash \Omega_{\beta}\right)$. We already have $S u \in\left(E_{\phi}\left([0, T], \mathbb{R}^{N}\right)\right)^{n}$ (Theorem 4.2). Moreover, in view of (H9) and (H10), it follows that

$$
\begin{aligned}
\theta_{i}\left(S_{i} u\right)(t)=\int_{0}^{t} g_{i}(t, s) \theta_{i} f_{i}(s, u(s)) d s & \geq 0, \\
\text { a.e. } t & \in[0, T], 1 \leq i \leq n .
\end{aligned}
$$

Since $u \in C_{\gamma} \cap\left(\bar{\Omega}_{\alpha} \backslash \Omega_{\beta}\right)$, there exists $R \in[\beta, \alpha]$ such that

$$
\begin{gathered}
\|u\|_{\phi, T}=R \quad \text { and } \quad \theta_{i} u_{i}(t) \geq \gamma(t) R>0, \\
\text { a.e. } t \in[0, T], 1 \leq i \leq n .
\end{gathered}
$$

It follows from (H9) and (H10) that for a.e. $t \in[0, T]$ and $1 \leq i \leq n$,

$$
\begin{aligned}
& \theta_{i}\left(S_{i} u\right)(t) \\
& \quad \geq \int_{0}^{t} g_{i}(t, s) \theta_{i} f_{i}\left(s, \theta_{1} R \gamma(s), \theta_{2} R \gamma(s), \cdots, \theta_{n} R \gamma(s)\right) d s .
\end{aligned}
$$

On the other hand, using (H4) $\left.\right|_{r=R}$, (4.13) and (4.14) $\left.\right|_{M_{0}=R}$, we find for $1 \leq i \leq n$,

$$
\begin{aligned}
\left|S_{i} u\right|_{\phi, T} & =\left|\int_{0}^{t} g_{i}(t, s) f_{i}(s, u(s)) d s\right|_{\phi, T} \\
& \leq\left|\int_{0}^{t} g_{i}(t, s)\left\{\eta_{R, i}(s)+K_{R, i} Q^{-1}\left(\phi\left(\frac{\left|u_{i}\right|}{R}\right)\right)\right\} d s\right|_{\phi, T} \\
& \leq\left|q_{i}\right|_{\phi, T}\left\{\left|\eta_{R, i}\right|_{Q, T}+K_{R, i}\left|Q^{-1}\left(\phi\left(\frac{\left|u_{i}\right|}{R}\right)\right)\right|_{Q, T}\right\} \\
& \leq\left|q_{i}\right|_{\phi, T}\left\{\left|\eta_{R, i}\right|_{Q, T}+K_{R, i}\left[1+\int_{0}^{T} \phi\left(\frac{\left|u_{i}(s)\right|}{R}\right) d s\right]\right\} .
\end{aligned}
$$

Now, we employ Lemma 9.2 in [14] to get 


$$
\int_{0}^{T} \phi\left(\frac{\left|u_{i}(s)\right|}{R}\right) d s \leq \frac{\left|u_{i}\right|_{\phi, T}}{R} \leq 1 .
$$

Hence, from (4.27) we get

$$
\left|S_{i} u\right|_{\phi, T} \leq\left|q_{i}\right|_{\phi, T}\left(\left|\eta_{R, i}\right|_{Q, T}+2 K_{R, i}\right), 1 \leq i \leq n
$$

which implies

$$
\|S u\|_{\phi, T} \leq \max _{1 \leq j \leq n}\left|q_{j}\right|_{\phi, T}\left(\left|\eta_{R, j}\right|_{Q, T}+2 K_{R, j}\right) .
$$

Now, using (4.29) in (4.26) yields

$$
\begin{aligned}
\theta_{i}\left(S_{i} u\right)(t) & \geq \frac{\int_{0}^{t} g_{i}(t, s) \theta_{i} f_{i}\left(s, \theta_{1} R \gamma(s), \theta_{2} R \gamma(s), \cdots, \theta_{n} R \gamma(s)\right) d s}{\max _{1 \leq j \leq n}\left|q_{j}\right|_{\phi, T}\left(\left|\eta_{R, j}\right|_{Q, T}+2 K_{R, j}\right)}\|S u\|_{\phi, T} \\
& \geq \gamma(t)\|S u\|_{\phi, T}, \quad \text { a.e. } t \in[0, T], \quad 1 \leq i \leq n
\end{aligned}
$$

where the last inequality follows from (H11). This completes the proof.

口

We are now ready to apply Theorem 2.2 to get the existence of a constant-sign solution in $\left(E_{\phi}\left([0, T], \mathbb{R}^{N}\right)\right)^{n}$.

Theorem 4.5. Let $P$ and $Q$ be complementary $N$-functions. Let (H1)-(H4) and (H9)-(H11) hold. Assume

(H12) there exists $\delta>0$ such that $\phi\left(\frac{x}{y}\right) \geq \delta \frac{\phi(x)}{\phi(y)}$ for $x, y \geq 0$;

(H13) there exists $\alpha>0$ such that for each $1 \leq i \leq n$,

$$
\left|q_{i}\right|_{\phi, T}\left(\left|\eta_{\alpha, i}\right|_{Q, T}+2 K_{\alpha, i}\right) \leq \alpha ;
$$

(H14) there exists $\beta>0$ such that for each $1 \leq i \leq n$,

$$
\begin{gathered}
\phi^{-1}\left(\delta \int_{0}^{T} \phi\left(\int_{0}^{s} g_{i}(s, \tau) \theta_{i} f_{i}\left(\tau, \theta_{1} \beta \gamma(\tau), \theta_{2} \beta \gamma(\tau), \cdots, \theta_{n} \beta \gamma(\tau)\right) d \tau\right) d s\right) \\
\geq \beta
\end{gathered}
$$


(of course we also assume the above integral exists).

Then, the system (1.1) has at least one constant-sign solution $u \in$ $\left(E_{\phi}\left([0, T], \mathbb{R}^{N}\right)\right)^{n}$ such that

(a) $\alpha \leq\|u\|_{\phi, T} \leq \beta$ and $\theta_{i} u_{i}(t) \geq \gamma(t) \alpha$, a.e. $t \in[0, T], 1 \leq i \leq n$ if $\alpha<\beta$

(a) $\beta \leq\|u\|_{\phi, T} \leq \alpha$ and $\theta_{i} u_{i}(t) \geq \gamma(t) \beta$, a.e. $t \in[0, T], 1 \leq i \leq n$ if $\beta<\alpha$.

Proof. Without any loss of generality, let $\beta<\alpha$. Since $S: C_{\gamma} \cap$ $\left(\bar{\Omega}_{\alpha} \backslash \Omega_{\beta}\right) \rightarrow C_{\gamma}$ is continuous and completely continuous (Theorem 4.2 and Lemmas 4.1), it suffices to show that (i) $\|S u\|_{\phi, T} \leq\|u\|_{\phi, T}$ for $u \in C_{\gamma} \cap \partial \Omega_{\alpha}$, and (ii) $\|S u\|_{\phi, T} \geq\|u\|_{\phi, T}$ for $u \in C_{\gamma} \cap \partial \Omega_{\beta}$.

To prove (i), let $u \in C_{\gamma} \cap \partial \Omega_{\alpha}$. Then,

$$
\|u\|_{\phi, T}=\alpha \quad \text { and } \quad \theta_{i} u_{i}(t) \geq \gamma(t) \alpha>0, \quad \text { a.e. } t \in[0, T], 1 \leq i \leq n .
$$

As in the proof of Lemma 4.1, we obtain (4.29) $\left.\right|_{R=\alpha}$, which, together with (H13) yields

$$
\|S u\|_{\phi, T} \leq \max _{1 \leq j \leq n}\left|q_{j}\right|_{\phi, T}\left(\left|\eta_{\alpha, j}\right|_{Q, T}+2 K_{\alpha, j}\right) \leq \alpha=\|u\|_{\phi, T}
$$

Next, we shall verify (ii). Let $u \in C_{\gamma} \cap \partial \Omega_{\beta}$. Then,

$$
\|u\|_{\phi, T}=\beta \quad \text { and } \quad \theta_{i} u_{i}(t) \geq \gamma(t) \beta>0 \text {, a.e. } t \in[0, T], 1 \leq i \leq n .
$$

Fix $1 \leq i \leq n$. As in the proof of Lemma 4.1 , we get (4.26) $\left.\right|_{R=\beta}$. Since $\phi(x)$ is an increasing function of $x$ for $x \geq 0$, it follows that

$$
\begin{aligned}
& \text { (4.30) } \quad \phi\left(\left|S_{i} u(t)\right|\right) \\
& \geq \phi\left(\int_{0}^{t} g_{i}(t, s) \theta_{i} f_{i}\left(s, \theta_{1} \beta \gamma(s), \theta_{2} \beta \gamma(s), \cdots, \theta_{n} \beta \gamma(s)\right) d s\right) \text {, a.e. } t \in[0, T] .
\end{aligned}
$$

Now, applying Lemma 9.2 in [14] gives 


$$
\int_{0}^{T} \phi\left(\frac{\left|S_{i} u(s)\right|}{\|S u\|_{\phi, T}}\right) d s \leq \frac{\left|S_{i} u\right|_{\phi, T}}{\|S u\|_{\phi, T}} \leq 1 .
$$

Also, using (H12) we find

$$
\int_{0}^{T} \phi\left(\frac{\left|S_{i} u(s)\right|}{\|S u\|_{\phi, T}}\right) d s \geq \delta \int_{0}^{T} \frac{\phi\left(\left|S_{i} u(s)\right|\right)}{\phi\left(\|S u\|_{\phi, T}\right)} d s .
$$

Coupling (4.31) and (4.32) leads to

$$
1 \geq \delta \int_{0}^{T} \frac{\phi\left(\left|S_{i} u(s)\right|\right)}{\phi\left(\|S u\|_{\phi, T}\right)} d s
$$

which, together with (4.30), provides

$$
\begin{gathered}
\phi\left(\|S u\|_{\phi, T}\right) \geq \delta \int_{0}^{T} \phi\left(\left|S_{i} u(s)\right|\right) d s \\
\geq \delta \int_{0}^{T} \phi\left(\int_{0}^{s} g_{i}(s, \tau) \theta_{i} f_{i}\left(\tau, \theta_{1} \beta \gamma(\tau), \theta_{2} \beta \gamma(\tau), \cdots, \theta_{n} \beta \gamma(\tau)\right) d \tau\right) d s .
\end{gathered}
$$

In view of (H14), it follows that

$$
\begin{gathered}
\|S u\|_{\phi, T} \\
\geq \phi^{-1}\left(\delta \int_{0}^{T} \phi\left(\int_{0}^{s} g_{i}(s, \tau) \theta_{i} f_{i}\left(\tau, \theta_{1} \beta \gamma(\tau), \theta_{2} \beta \gamma(\tau), \cdots, \theta_{n} \beta \gamma(\tau)\right) d \tau\right) d s\right) \\
\geq \beta=\|u\|_{\phi, T} .
\end{gathered}
$$

Having established (i) and (ii), we conclude from Theorem 2.2 that $S$ has a fixed point $u \in C_{\gamma} \cap\left(\bar{\Omega}_{\alpha} \backslash \Omega_{\beta}\right)$. Therefore, conclusion (b) follows immediately.

Remark 4.6. In (H13) if we have strict inequality instead, i.e., condition $(\mathrm{H} 8)^{\prime}$, then from the proof of Theorem 4.5 we see that a fixed point $u$ of $S$ must satisfy $\|u\|_{\phi, T} \neq \alpha$. Likewise, if the inequality in (H14) is strict, i.e., 
$\phi^{-1}\left(\delta \int_{0}^{T} \phi\left(\int_{0}^{s} g_{i}(s, \tau) \theta_{i} f_{i}\left(\tau, \theta_{1} \beta \gamma(\tau), \theta_{2} \beta \gamma(\tau), \cdots, \theta_{n} \beta \gamma(\tau)\right) d \tau\right) d s\right)>\beta$,

then a fixed point $u$ of $S$ must fulfill $\|u\|_{\phi, T} \neq \beta$. Hence, with strict inequalities in (H13) and (H14), the conclusion of Theorem 4.5 becomes: the system (1.1) has at least one constant-sign solution $u \in\left(E_{\phi}\left([0, T], \mathbb{R}^{N}\right)\right)^{n}$ such that

(a) $\alpha<\|u\|_{\phi, T}<\beta$ and $\theta_{i} u_{i}(t)>\gamma(t) \alpha$, a.e. $t \in[0, T], 1 \leq i \leq n$ if $\alpha<\beta$;

(b) $\beta<\|u\|_{\phi, T}<\alpha$ and $\theta_{i} u_{i}(t)>\gamma(t) \beta, \quad$ a.e. $t \in[0, T], 1 \leq i \leq n$ if $\beta<\alpha$.

Our next two result gives the existence of multiple constant-sign solutions of $(1.1)$ in $\left(E_{\phi}\left([0, T], \mathbb{R}^{N}\right)\right)^{n}$.

Theorem 4.6. Let $P$ and $Q$ be complementary $N$-functions. Assume (H1)-(H6) and (H9)-(H12) hold. Let (H7)' be satisfied for $\alpha=\alpha_{0}$, (H13) be satisfied for $\alpha=\alpha_{\ell}, \ell=1,2, \cdots, k$, and (H14) be satisfied for $\beta=\beta_{\ell}, \ell=1,2, \cdots, m$.

(a) If $m=k+1$ and $0<\beta_{1}<\alpha_{1}<\cdots<\beta_{k}<\alpha_{k}<$ $\beta_{k+1}$, then (1.1) has (at least) $2 k$ constant-sign solutions $u^{1}, \cdots, u^{2 k} \in$ $\left(E_{\phi}\left([0, T], \mathbb{R}^{N}\right)\right)^{n}$ such that

$$
\beta_{1} \leq\left\|u^{1}\right\|_{\phi, T} \leq \alpha_{1} \leq\left\|u^{2}\right\|_{\phi, T} \leq \beta_{2} \leq \cdots \leq \alpha_{k} \leq\left\|u^{2 k}\right\|_{\phi, T} \leq \beta_{k+1} .
$$

(b) If $m=k$ and $0<\beta_{1}<\alpha_{1}<\cdots<\beta_{k}<\alpha_{k}$, then (1.1) has (at least) $2 k-1$ constant-sign solutions $u^{1}, \cdots, u^{2 k-1} \in\left(E_{\phi}\left([0, T], \mathbb{R}^{N}\right)\right)^{n}$ such that

$$
\beta_{1} \leq\left\|u^{1}\right\|_{\phi, T} \leq \alpha_{1} \leq\left\|u^{2}\right\|_{\phi, T} \leq \beta_{2} \leq \cdots \leq \beta_{k} \leq\left\|u^{2 k-1}\right\|_{\phi, T} \leq \alpha_{k} .
$$

(c) If $m=k+1$ and $0<\alpha_{0}<\beta_{1}<\alpha_{1}<\cdots<\alpha_{m-1}<\beta_{m}$, then (1.1) has (at least) $2 m$ constant-sign solutions $u^{0}, \cdots, u^{2 m-1} \in$ $\left(E_{\phi}\left([0, T], \mathbb{R}^{N}\right)\right)^{n}$ such that 


$$
\begin{aligned}
0 & \leq\left\|u^{0}\right\|_{\phi, T}<\alpha_{0}<\left\|u^{1}\right\|_{\phi, T} \leq \beta_{1} \leq\left\|u^{2}\right\|_{\phi, T} \\
& \leq \alpha_{1} \leq \cdots \leq \alpha_{m-1} \leq\left\|u^{2 m-1}\right\|_{\phi, T} \leq \beta_{m} .
\end{aligned}
$$

(d) If $m=k$ and $0<\alpha_{0}<\beta_{1}<\alpha_{1}<\cdots<\beta_{m}<\alpha_{m}$, then (1.1) has (at least) $2 m+1$ constant-sign solutions $u^{0}, \cdots, u^{2 m} \in$ $\left(E_{\phi}\left([0, T], \mathbb{R}^{N}\right)\right)^{n}$ such that

$$
\begin{gathered}
0 \leq\left\|u^{0}\right\|_{\phi, T}<\alpha_{0}<\left\|u^{1}\right\|_{\phi, T} \leq \beta_{1} \leq\left\|u^{2}\right\|_{\phi, T} \\
\leq \alpha_{1} \leq \cdots \leq \beta_{m} \leq\left\|u^{2 m}\right\|_{\phi, T} \leq \alpha_{m} .
\end{gathered}
$$

Proof. In (a) and (b), we just apply Theorem 4.5 repeatedly. In (c) and (d), Theorem 4.3 (and Remark 4.5) is used to obtain the existence of $u^{0} \in\left(E_{\phi}\left([0, T], \mathbb{R}^{N}\right)\right)^{n}$ with $0 \leq\left\|u^{0}\right\|_{\phi, T}<\alpha_{0}$, the results then follow by repeated use of Theorem 4.5.

Theorem 4.7. Let $P$ and $Q$ be complementary $N$-functions. Assume (H1)-(H4) and (H9)-(H12) hold. Let (H8)' be satisfied for $\alpha=\alpha_{0}$, (H13) be satisfied for $\alpha=\alpha_{\ell}, \ell=1,2, \cdots, k$, and (H14) be satisfied for $\beta=\beta_{\ell}, \ell=1,2, \cdots, m$. Then, the conclusions (a)-(d) of Theorem 4.6 hold.

Proof. The proof is similar to that of Theorem 4.6, with an application of Theorem 4.4 instead of Theorem 4.3.

Remark 4.7. Suppose in Theorems 4.6 and 4.7 we have some strict inequalities in (H13) and (H14), say, involving $\alpha_{i}$ and $\beta_{j}$ for some $i \in\{1,2, \cdots, k\}$ and some $j \in\{1,2, \cdots, m\}$. Then, noting Remark 4.6, those inequalities in the conclusion involving $\alpha_{i}$ and $\beta_{j}$ will also be strict.

\section{REFERENCES}

1. R. P. Agarwal, D. O'Regan and P. J. Y. Wong, Positive Solutions of Differential, Difference and Integral Equations, Kluwer, Dordrecht, (1999). 
2. R. P. Agarwal, D. O'Regan and P. J. Y. Wong, Constant-sign solutions of a system of Fredholm integral equations, Acta Applicandae Mathematicae 80 (2004), 57-94.

3. R. P. Agarwal, D. O'Regan and P. J. Y. Wong, Eigenvalues of a system of Fredholm integral equations, Math. Comput. Modelling 39 (2004), 1113-1150.

4. R. P. Agarwal, D. O'Regan and P. J. Y. Wong, Triple solutions of constant sign for a system of Fredholm integral equations, Cubo 6 (2004), 1-45.

5. R. P. Agarwal, D. O'Regan and P. J. Y. Wong, Constant-sign $L^{p}$ solutions for a system of integral equations, Results in Mathematics 46 (2004), 195-219.

6. R. P. Agarwal, D. O'Regan and P. J. Y. Wong, Solutions of a system of integral equations in Orlicz spaces, preprint.

7. R. P. Agarwal, D. O'Regan and P. J. Y. Wong, Constant-sign solutions of a system of Volterra integral equations, Comput. Math. Appl., to appear.

8. P. J. Bushell and W. Okrasiński, Uniqueness of solutions for a class of nonlinear Volterra integral equations with convolution kernel, Math. Proc. Cambridge Philos. Soc. 106 (1989), 547-552.

9. P. J. Bushell and W. Okrasiński, Nonlinear Volterra integral equations with convolution kernel, J. London Math. Soc. 41 (1990), 503-510.

10. C. Corduneanu, Integral Equations and Stability of Feedback Systems, Academic Press, New York, (1973).

11. C. Corduneanu, Integral Equations and Applications, Cambridge Univ. Press, New York, (1990).

12. M. A. Krasnosel'skii, Positive Solutions of Operator Equations, Noordhoff, Groningen, (1964).

13. M. A. Krasnosel'skii, Topological Methods in the Theory of Nonlinear Integral Equations, Pergamon Press, Oxford, (1964).

14. M. A. Krasnosel'skii and Ya. B. Rutickii, Convex Functions and Orlicz Spaces (Trans. L. F. Boron), Noordhoff, Groningen, (1961).

15. M. Meehan and D. O'Regan, Positive solutions of Volterra integral equations using integral inequalities, J. Inequal. Appl. 7 (2002), 285-307.

16. D. O'Regan, Solutions in Orlicz spaces to Uryson integral equations, Proc. Royal Irish Acad 96A (1996), 67-78.

17. D. O'Regan, A topological approach to integral inclusions, Proc Royal Irish Acad 97A (1997), 101-111.

18. D. O'Regan and M. Meehan, Existence Theory for Nonlinear Integral and Integrodifferential Equations, Kluwer, Dordrecht, (1998).

19. W. Orlicz and S. Szufla, On the structure of $L_{\phi}$-solution sets of integral equations in Banach spaces, Studia Math. 77 (1984), 465-477.

20. M. M. Rao and Z. D. Ren, Theory of Orlicz Spaces, Marcel Dekker, New York, (1991).

21. R. L. Wheeden and A. Zygmund, Measure and Integral, Monographs and Textbooks in Pure and Applied Mathematics, Marcel Dekker Inc., New York and Basel, (1977). 
Department of Mathematical Sciences, Florida Institute of Technology, Melbourne, Florida 32901-6975

Email address: agarwal@fit.edu

Department of Mathematics, National University of Ireland, Galway, IRELAND

Email address: donal.oregan@nuigalway.ie

School of Electrical and Electronic Engineering, Nanyang Technological University, 50 Nanyang Avenue, Singapore 639798, Singapore

Email address: ejywong@ntu.edu.sg 